\title{
Composition operators on Hardy spaces
}

\author{
Episode I
}

VI Curso Internacional de Análisis Matemático en Andalucía

Antequera septiembre 2014

\author{
Pascal Lefèvre
}

Université d'Artois, France 
(1) Lecture 1

- Classical Hardy spaces on $\mathbb{D}$

- Composition operators

- Boundedness

- Compactness 


\section{Program}

(1) Lecture 1

- Classical Hardy spaces on $\mathbb{D}$

- Composition operators

- Boundedness

- Compactness

(2) Lecture 2

- $H^{\infty}$

- Hardy-Orlicz spaces and their composition operators

- Carleson versus Nevanlinna 


\section{Program}

(1) Lecture 1

- Classical Hardy spaces on $\mathbb{D}$

- Composition operators

- Boundedness

- Compactness

(2) Lecture 2

- $H^{\infty}$

- Hardy-Orlicz spaces and their composition operators

- Carleson versus Nevanlinna

(3) Lecture 3

- Schatten classes, approximation numbers

- Absolutely summing composition operators

- Some open problems... 


\section{Notations}

- $\mathbb{D}=\{z \in \mathbb{C}|| z \mid<1\}$ 


\section{Notations}

- $\mathbb{D}=\{z \in \mathbb{C}|| z \mid<1\}$

$\mathcal{A}$ is the normalized area measure on $\mathbb{D}$. 


\section{Notations}

- $\mathbb{D}=\{z \in \mathbb{C}|| z \mid<1\}$

$\mathcal{A}$ is the normalized area measure on $\mathbb{D}$.

- $\mathbb{T}=\{z \in \mathbb{C}|| z \mid=1\}=\partial \mathbb{D}=\mathbb{R} / \mathbb{Z}$ 


\section{Notations}

- $\mathbb{D}=\{z \in \mathbb{C}|| z \mid<1\}$

$\mathcal{A}$ is the normalized area measure on $\mathbb{D}$.

- $\mathbb{T}=\{z \in \mathbb{C}|| z \mid=1\}=\partial \mathbb{D}=\mathbb{R} / \mathbb{Z}$

$\lambda$ is the Haar measure on $\mathbb{T}$. 


\section{Notations}

- $\mathbb{D}=\{z \in \mathbb{C}|| z \mid<1\}$

$\mathcal{A}$ is the normalized area measure on $\mathbb{D}$.

- $\mathbb{T}=\{z \in \mathbb{C}|| z \mid=1\}=\partial \mathbb{D}=\mathbb{R} / \mathbb{Z}$

$\lambda$ is the Haar measure on $\mathbb{T}$.

- $p \in[1,+\infty]$ 


\section{Classical Hardy spaces on the unit disk}

$$
\frac{p \in[1,+\infty):}{\text { and }} \quad H^{p}=\left\{\left.f \in \mathcal{H}(\mathbb{D})\left|\sup _{r<1} \int_{\mathbb{T}}\right| f(r z)\right|^{p} d \lambda<\infty\right\}
$$




\section{Classical Hardy spaces on the unit disk}

$$
\begin{aligned}
\frac{p \in[1,+\infty):}{\text { and }} & H^{p}=\left\{\left.f \in \mathcal{H}(\mathbb{D})\left|\sup _{r<1} \int_{\mathbb{T}}\right| f(r z)\right|^{p} d \lambda<\infty\right\} \\
\|f\|_{p} & =\sup _{r<1}\left(\int_{\mathbb{T}}|f(r z)|^{p} d \lambda\right)^{1 / p}
\end{aligned}
$$




\section{Classical Hardy spaces on the unit disk}

$\begin{aligned} & \frac{p \in[1,+\infty):}{\text { and }} H^{p}=\left\{\left.f \in \mathcal{H}(\mathbb{D})\left|\sup _{r<1} \int_{\mathbb{T}}\right| f(r z)\right|^{p} d \lambda<\infty\right\} \\ &\|f\|_{p}=\sup _{r<1}\left(\int_{\mathbb{T}}|f(r z)|^{p} d \lambda\right)^{1 / p}=\sup _{r<1}\left\|f_{r}\right\|_{L^{p}(\mathbb{T})}\end{aligned}$

where $f_{r}(z)=f(r z)$ with $r \in(0,1)$ and $z \in \overline{\mathbb{D}}$. 


\section{Classical Hardy spaces on the unit disk}

- $p \in[1,+\infty): \quad H^{p}=\left\{\left.f \in \mathcal{H}(\mathbb{D})\left|\sup _{r<1} \int_{\mathbb{T}}\right| f(r z)\right|^{p} d \lambda<\infty\right\}$ and

$$
\|f\|_{p}=\sup _{r<1}\left(\int_{\mathbb{T}}|f(r z)|^{p} d \lambda\right)^{1 / p}=\sup _{r<1}\left\|f_{r}\right\|_{L^{p}(\mathbb{T})}
$$

where $f_{r}(z)=f(r z)$ with $r \in(0,1)$ and $z \in \overline{\mathbb{D}}$.

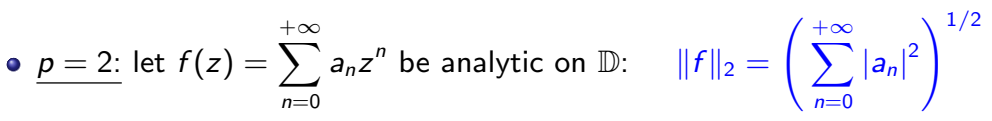




\section{Classical Hardy spaces on the unit disk}

$\underline{p \in[1,+\infty):} \quad H^{p}=\left\{\left.f \in \mathcal{H}(\mathbb{D})\left|\sup _{r<1} \int_{\mathbb{T}}\right| f(r z)\right|^{p} d \lambda<\infty\right\}$ and

$$
\|f\|_{p}=\sup _{r<1}\left(\int_{\mathbb{T}}|f(r z)|^{p} d \lambda\right)^{1 / p}=\sup _{r<1}\left\|f_{r}\right\|_{L^{p}(\mathbb{T})}
$$

where $f_{r}(z)=f(r z)$ with $r \in(0,1)$ and $z \in \overline{\mathbb{D}}$.

- $\underline{p=2:}$ let $f(z)=\sum_{n=0}^{+\infty} a_{n} z^{n}$ be analytic on $\mathbb{D}: \quad\|f\|_{2}=\left(\sum_{n=0}^{+\infty}\left|a_{n}\right|^{2}\right)^{1 / 2}$

- $p=+\infty$ : the space of bounded analytic functions on $\mathbb{D}$ :

$$
H^{\infty}=\left\{f \in \mathcal{H}(\mathbb{D})\left|\|f\|_{\infty}=\sup _{z \in \mathbb{D}}\right| f(z) \mid<\infty\right\}
$$




\section{Classical Hardy spaces on the unit disk}

- $\underline{p \in[1,+\infty):} \quad H^{p}=\left\{\left.f \in \mathcal{H}(\mathbb{D})\left|\sup _{r<1} \int_{\mathbb{T}}\right| f(r z)\right|^{p} d \lambda<\infty\right\}$ and

$$
\|f\|_{p}=\sup _{r<1}\left(\int_{\mathbb{T}}|f(r z)|^{p} d \lambda\right)^{1 / p}=\sup _{r<1}\left\|f_{r}\right\|_{L^{p}(\mathbb{T})}
$$

where $f_{r}(z)=f(r z)$ with $r \in(0,1)$ and $z \in \overline{\mathbb{D}}$.

- $\underline{p=2}$ : let $f(z)=\sum_{n=0}^{+\infty} a_{n} z^{n}$ be analytic on $\mathbb{D}: \quad\|f\|_{2}=\left(\sum_{n=0}^{+\infty}\left|a_{n}\right|^{2}\right)^{1 / 2}$

- $p=+\infty$ : the space of bounded analytic functions on $\mathbb{D}$ :

$$
H^{\infty}=\left\{f \in \mathcal{H}(\mathbb{D})\left|\|f\|_{\infty}=\sup _{z \in \mathbb{D}}\right| f(z) \mid<\infty\right\}
$$

They are all Banach spaces... 


\section{Some (useful) properties...}

- Every $f \in H^{p}$ has almost everywhere radial limit $f^{*}$

$$
f^{*}\left(e^{i t}\right)=\lim _{r \rightarrow 1^{-}} f\left(r e^{i t}\right) .
$$

It is known that $f^{*} \in L^{p}(\mathbb{T})$ and $\|f\|_{H^{p}}=\left\|f^{*}\right\|_{L^{p}(\mathbb{T})}$.

In fact, $f^{*} \in\left\{g \in L^{p}(\mathbb{T}) \mid \hat{g}(m)=0\right.$ for every $\left.m<0\right\}$. 


\section{Some (useful) properties...}

- Every $f \in H^{p}$ has almost everywhere radial limit $f^{*}$

$$
f^{*}\left(e^{i t}\right)=\lim _{r \rightarrow 1^{-}} f\left(r e^{i t}\right) .
$$

It is known that $f^{*} \in L^{p}(\mathbb{T})$ and $\|f\|_{H^{p}}=\left\|f^{*}\right\|_{L^{p}(\mathbb{T})}$.

In fact, $f^{*} \in\left\{g \in L^{p}(\mathbb{T}) \mid \hat{g}(m)=0\right.$ for every $\left.m<0\right\}$.

- Conversely, if $g \in L^{p}(\mathbb{T})$, with $\hat{g}(m)=0$ for every $m<0$, the Poisson integral of $g$ at point $z=r e^{2 i \pi \theta}$

$$
P[g](z)=P_{r} * g(\theta)=\int_{0}^{1} P_{r}(\theta-t) g\left(e^{2 i \pi t}\right) d t,
$$

belongs to $H^{p}$. Moreover $(P[g])^{*}=g$. 


\section{Some (useful) properties...}

- Every $f \in H^{p}$ has almost everywhere radial limit $f^{*}$

$$
f^{*}\left(e^{i t}\right)=\lim _{r \rightarrow 1^{-}} f\left(r e^{i t}\right) .
$$

It is known that $f^{*} \in L^{p}(\mathbb{T})$ and $\|f\|_{H^{p}}=\left\|f^{*}\right\|_{L^{p}(\mathbb{T})}$.

In fact, $f^{*} \in\left\{g \in L^{p}(\mathbb{T}) \mid \hat{g}(m)=0\right.$ for every $\left.m<0\right\}$.

- Conversely, if $g \in L^{p}(\mathbb{T})$, with $\hat{g}(m)=0$ for every $m<0$, the Poisson integral of $g$ at point $z=r e^{2 i \pi \theta}$

$$
P[g](z)=P_{r} * g(\theta)=\int_{0}^{1} P_{r}(\theta-t) g\left(e^{2 i \pi t}\right) d t,
$$

belongs to $H^{P}$. Moreover $(P[g])^{*}=g$.

- We have $H^{p} \sim\left\{g \in L^{p}(\mathbb{T}) \mid \hat{g}(m)=0\right.$ for every $\left.m<0\right\}$. 


\section{Some (useful) properties...}

- Every $f \in H^{p}$ has almost everywhere radial limit $f^{*}$

$$
f^{*}\left(e^{i t}\right)=\lim _{r \rightarrow 1^{-}} f\left(r e^{i t}\right) .
$$

It is known that $f^{*} \in L^{p}(\mathbb{T})$ and $\|f\|_{H^{p}}=\left\|f^{*}\right\|_{L^{p}(\mathbb{T})}$.

In fact, $f^{*} \in\left\{g \in L^{p}(\mathbb{T}) \mid \hat{g}(m)=0\right.$ for every $\left.m<0\right\}$.

- Conversely, if $g \in L^{p}(\mathbb{T})$, with $\hat{g}(m)=0$ for every $m<0$, the Poisson integral of $g$ at point $z=r e^{2 i \pi \theta}$

$$
P[g](z)=P_{r} * g(\theta)=\int_{0}^{1} P_{r}(\theta-t) g\left(e^{2 i \pi t}\right) d t,
$$

belongs to $H^{p}$. Moreover $(P[g])^{*}=g$.

- We have $H^{p} \sim\left\{g \in L^{p}(\mathbb{T}) \mid \hat{g}(m)=0\right.$ for every $\left.m<0\right\}$.

- Hence we will consider that a function $f \in H^{p}$ is defined, not only on $\mathbb{D}$, but on the whole $\overline{\mathbb{D}}=\mathbb{D} \cup \mathbb{T}$. 


\section{Some (useful) properties...}

- Factorization: we can write $f \in H^{p}$ as $f=B . g$ where $B$ is inner (i.e. $\left|B^{*}\right|=1$ a.e.) and $g$ does not vanish on $\mathbb{D}$. 


\section{Some (useful) properties...}

- Factorization: we can write $f \in H^{p}$ as $f=B . g$ where $B$ is inner (i.e. $\left|B^{*}\right|=1$ a.e.) and $g$ does not vanish on $\mathbb{D}$. Since $\left|f^{*}\right|=\left|g^{*}\right|($ a.e. on $\mathbb{T})$, we have $\|f\|_{H^{p}}=\|g\|_{H^{p}}$. 


\section{Some (useful) properties...}

- Factorization: we can write $f \in H^{p}$ as $f=B . g$ where $B$ is inner (i.e. $\left|B^{*}\right|=1$ a.e.) and $g$ does not vanish on $\mathbb{D}$. Since $\left|f^{*}\right|=\left|g^{*}\right|($ a.e. on $\mathbb{T})$, we have $\|f\|_{H^{p}}=\|g\|_{H^{p}}$.

- For every $z \in \mathbb{D}$, the point evaluation at $z \in \mathbb{D}$, is defined on $H^{p}$ by

$$
\delta_{z}(f)=f(z) .
$$

$\delta_{z}$ is a continuous linear functional and 


\section{Some (useful) properties...}

- Factorization: we can write $f \in H^{p}$ as $f=B . g$ where $B$ is inner (i.e. $\left|B^{*}\right|=1$ a.e.) and $g$ does not vanish on $\mathbb{D}$. Since $\left|f^{*}\right|=\left|g^{*}\right|($ a.e. on $\mathbb{T})$, we have $\|f\|_{H^{p}}=\|g\|_{H^{p}}$.

- For every $z \in \mathbb{D}$, the point evaluation at $z \in \mathbb{D}$, is defined on $H^{p}$ by

$$
\delta_{z}(f)=f(z) .
$$

$\delta_{z}$ is a continuous linear functional and

- On the Hilbert space $H^{2}$, the functional $\delta_{z}$ is associated to the reproducing kernel $w \in \overline{\mathbb{D}} \longmapsto \frac{1}{1-\bar{z} w}$.

- $\left\|\delta_{z}\right\|_{\left(H^{p}\right)^{*}}=\left(\frac{1}{1-|z|^{2}}\right)^{1 / p} \approx \frac{1}{(1-|z|)^{1 / p}}$. 


\section{Some (useful) properties...}

Indeed: on $H^{2}$, we want to estimate $\sup _{\sum\left|a_{n}\right|^{2}=1}\left|\sum_{n=0}^{+\infty} a_{n} z^{n}\right|$ which is clearly 


\section{Some (useful) properties...}

Indeed: on $H^{2}$, we want to estimate $\sup _{\sum\left|a_{n}\right|^{2}=1}\left|\sum_{n=0}^{+\infty} a_{n} z^{n}\right|$ which is clearly

$$
\left(\sum_{n=0}^{+\infty}\left|z^{n}\right|^{2}\right)^{1 / 2}=\left(\frac{1}{1-|z|^{2}}\right)^{1 / 2}
$$




\section{Some (useful) properties...}

Indeed: on $H^{2}$, we want to estimate $\sup _{\sum\left|a_{n}\right|^{2}=1}\left|\sum_{n=0}^{+\infty} a_{n} z^{n}\right|$ which is clearly

$$
\left(\sum_{n=0}^{+\infty}\left|z^{n}\right|^{2}\right)^{1 / 2}=\left(\frac{1}{1-|z|^{2}}\right)^{1 / 2}
$$

Now for $p \neq 2$, let us write $f \in H^{p}$ as $f=B$.g where $B$ is inner and $g$ does not vanish on $\mathbb{D}$. 


\section{Some (useful) properties...}

Indeed: on $H^{2}$, we want to estimate $\sup _{\sum\left|a_{n}\right|^{2}=1}\left|\sum_{n=0}^{+\infty} a_{n} z^{n}\right|$ which is clearly

$$
\left(\sum_{n=0}^{+\infty}\left|z^{n}\right|^{2}\right)^{1 / 2}=\left(\frac{1}{1-|z|^{2}}\right)^{1 / 2}
$$

Now for $p \neq 2$, let us write $f \in H^{p}$ as $f=B$.g where $B$ is inner and $g$ does not vanish on $\mathbb{D}$.

Hence,

$$
\left|\delta_{z}(f)\right|^{p}=|f(z)|^{p} \leq\left|g^{p / 2}(z)\right|^{2} \leq\left\|\delta_{z}\right\|_{\left(H^{2}\right)^{*}}^{2}\left\|g^{p / 2}\right\|_{H^{2}}^{2}=\frac{\|g\|_{H^{p}}^{p}}{1-|z|^{2}} .
$$

so 


\section{Some (useful) properties...}

Indeed: on $H^{2}$, we want to estimate $\sup _{\sum\left|a_{n}\right|^{2}=1}\left|\sum_{n=0}^{+\infty} a_{n} z^{n}\right|$ which is clearly

$$
\left(\sum_{n=0}^{+\infty}\left|z^{n}\right|^{2}\right)^{1 / 2}=\left(\frac{1}{1-|z|^{2}}\right)^{1 / 2}
$$

Now for $p \neq 2$, let us write $f \in H^{p}$ as $f=B$.g where $B$ is inner and $g$ does not vanish on $\mathbb{D}$.

Hence,

$$
\left|\delta_{z}(f)\right|^{p}=|f(z)|^{p} \leq\left|g^{p / 2}(z)\right|^{2} \leq\left\|\delta_{z}\right\|_{\left(H^{2}\right)^{*}}^{2}\left\|g^{p / 2}\right\|_{H^{2}}^{2}=\frac{\|g\|_{H^{p}}^{p}}{1-|z|^{2}} .
$$

so

$$
\left\|\delta_{z}\right\|_{\left(H^{p}\right)^{*}} \leq\left(\frac{1}{1-|z|^{2}}\right)^{1 / p}
$$




\section{Some (useful) properties...}

Indeed: on $H^{2}$, we want to estimate $\sup _{\sum\left|a_{n}\right|^{2}=1}\left|\sum_{n=0}^{+\infty} a_{n} z^{n}\right|$ which is clearly

$$
\left(\sum_{n=0}^{+\infty}\left|z^{n}\right|^{2}\right)^{1 / 2}=\left(\frac{1}{1-|z|^{2}}\right)^{1 / 2}
$$

Now for $p \neq 2$, let us write $f \in H^{p}$ as $f=B$.g where $B$ is inner and $g$ does not vanish on $\mathbb{D}$.

Hence,

$$
\left|\delta_{z}(f)\right|^{p}=|f(z)|^{p} \leq\left|g^{p / 2}(z)\right|^{2} \leq\left\|\delta_{z}\right\|_{\left(H^{2}\right)^{*}}^{2}\left\|g^{p / 2}\right\|_{H^{2}}^{2}=\frac{\|g\|_{H^{p}}^{p}}{1-|z|^{2}} .
$$

so

$$
\left\|\delta_{z}\right\|_{\left(H^{P}\right)^{*}} \leq\left(\frac{1}{1-|z|^{2}}\right)^{1 / p}
$$

For the reverse inequality: consider $w \in \overline{\mathbb{D}} \mapsto\left(\frac{1}{1-\bar{z} w}\right)^{2 / p}$.

\section{Curso Internacional de Análisis Matemático en Andalucía}




\section{Composition operators}

They are the operators of type: $\quad C_{\varphi}: f \longrightarrow f \circ \varphi \quad$ where $\varphi: \mathbb{D} \rightarrow \mathbb{D}$ is analytic.

A few natural questions: 


\section{Composition operators}

They are the operators of type: $\quad C_{\varphi}: f \longrightarrow f \circ \varphi \quad$ where $\varphi: \mathbb{D} \rightarrow \mathbb{D}$ is analytic.

A few natural questions:

- When is it bounded? 


\section{Composition operators}

They are the operators of type: $\quad C_{\varphi}: f \longrightarrow f \circ \varphi \quad$ where $\varphi: \mathbb{D} \rightarrow \mathbb{D}$ is analytic.

A few natural questions:

- When is it bounded?

- When is it compact? 


\section{Composition operators}

They are the operators of type: $\quad C_{\varphi}: f \longrightarrow f \circ \varphi \quad$ where $\varphi: \mathbb{D} \rightarrow \mathbb{D}$ is analytic.

A few natural questions:

- When is it bounded?

- When is it compact?

- When is it very compact? 


\section{Composition operators}

They are the operators of type: $\quad C_{\varphi}: f \longrightarrow f \circ \varphi \quad$ where $\varphi: \mathbb{D} \rightarrow \mathbb{D}$ is analytic.

A few natural questions:

- When is it bounded?

- When is it compact?

- When is it very compact?

- Understand the link: "Operator $C_{\varphi}$ " $\stackrel{? ?}{\longleftrightarrow}$ "symbol $\varphi$ " 


\section{Composition operators}

They are the operators of type: $\quad C_{\varphi}: f \longrightarrow f \circ \varphi \quad$ where $\varphi: \mathbb{D} \rightarrow \mathbb{D}$ is analytic.

A few natural questions:

- When is it bounded?

- When is it compact?

- When is it very compact?

- Understand the link: "Operator $C_{\varphi}$ " $\stackrel{? ?}{\longleftrightarrow}$ "symbol $\varphi$ "

The composition operators $C_{\varphi}: H^{p} \longrightarrow H^{p}$ are always bounded. 


\section{Composition operators}

They are the operators of type: $\quad C_{\varphi}: f \longrightarrow f \circ \varphi \quad$ where $\varphi: \mathbb{D} \rightarrow \mathbb{D}$ is analytic.

A few natural questions:

- When is it bounded?

- When is it compact?

- When is it very compact?

- Understand the link: "Operator $C_{\varphi}$ " $\stackrel{? ?}{\longleftrightarrow}$ "symbol $\varphi$ "

The composition operators $C_{\varphi}: H^{p} \longrightarrow H^{p}$ are always bounded.

There are several ways to see/understand this statement ! 


\section{Composition operators}

They are the operators of type: $\quad C_{\varphi}: f \longrightarrow f \circ \varphi \quad$ where $\varphi: \mathbb{D} \rightarrow \mathbb{D}$ is analytic.

A few natural questions:

- When is it bounded?

- When is it compact ?

- When is it very compact ?

- Understand the link: "Operator $C_{\varphi}$ " $\stackrel{? ?}{\longleftrightarrow}$ "symbol $\varphi$ "

The composition operators $C_{\varphi}: H^{p} \longrightarrow H^{p}$ are always bounded.

There are several ways to see/understand this statement !

( A simple hilbertian proof (cf Shapiro's monograph). 


\section{Composition operators}

They are the operators of type: $\quad C_{\varphi}: f \longrightarrow f \circ \varphi \quad$ where $\varphi: \mathbb{D} \rightarrow \mathbb{D}$ is analytic.

A few natural questions:

- When is it bounded?

- When is it compact ?

- When is it very compact ?

- Understand the link: "Operator $C_{\varphi}$ " $\stackrel{? ?}{\longleftrightarrow}$ "symbol $\varphi$ "

The composition operators $C_{\varphi}: H^{p} \longrightarrow H^{p}$ are always bounded.

There are several ways to see/understand this statement !

- A simple hilbertian proof (cf Shapiro's monograph).

(1) The Littlewood subordination principle. 


\section{Composition operators}

They are the operators of type: $\quad C_{\varphi}: f \longrightarrow f \circ \varphi \quad$ where $\varphi: \mathbb{D} \rightarrow \mathbb{D}$ is analytic.

A few natural questions:

- When is it bounded?

- When is it compact?

- When is it very compact ?

- Understand the link: "Operator $C_{\varphi}$ " $\stackrel{? ?}{\longleftrightarrow}$ "symbol $\varphi$ "

The composition operators $C_{\varphi}: H^{p} \longrightarrow H^{p}$ are always bounded.

There are several ways to see/understand this statement !

( A simple hilbertian proof (cf Shapiro's monograph).

(1) The Littlewood subordination principle.

(2) Using the Nevanlinna counting function $N_{\varphi}$. 


\section{Composition operators}

They are the operators of type: $\quad C_{\varphi}: f \longrightarrow f \circ \varphi \quad$ where $\varphi: \mathbb{D} \rightarrow \mathbb{D}$ is analytic.

A few natural questions:

- When is it bounded?

- When is it compact?

- When is it very compact ?

- Understand the link: "Operator $C_{\varphi}$ " $\stackrel{? ?}{\longleftrightarrow}$ "symbol $\varphi$ "

The composition operators $C_{\varphi}: H^{p} \longrightarrow H^{p}$ are always bounded.

There are several ways to see/understand this statement !

( A simple hilbertian proof (cf Shapiro's monograph).

(1) The Littlewood subordination principle.

(2) Using the Nevanlinna counting function $N_{\varphi}$.

(3) The Carleson embedding point of view, in terms of Carleson measures. 


\section{Boundedness}

Let us treat the case of the special (but important) case of Moebius transformations, which are automorphisms of the disk. Consider the Moebius transformation $q_{a}(z)=\frac{a-z}{1-\bar{a} z} \quad$, where $a \in \mathbb{D}$. 


\section{Boundedness}

Let us treat the case of the special (but important) case of Moebius transformations, which are automorphisms of the disk. Consider the Moebius transformation $q_{a}(z)=\frac{a-z}{1-\bar{a} z} \quad$, where $a \in \mathbb{D}$.

For every polynomial $f$, we have

$$
\left\|f \circ q_{a}\right\|_{H^{p}}^{p}=\int_{\mathbb{T}}|f(z)|^{p} \frac{1-|a|^{2}}{|1-\bar{a} z|^{2}} d \lambda \leq \frac{1+|a|}{1-|a|}\|f\|_{H^{p}}^{p} .
$$




\section{Boundedness}

Let us treat the case of the special (but important) case of Moebius transformations, which are automorphisms of the disk. Consider the Moebius transformation $q_{a}(z)=\frac{a-z}{1-\bar{a} z} \quad$, where $a \in \mathbb{D}$.

For every polynomial $f$, we have

$$
\left\|f \circ q_{a}\right\|_{H^{p}}^{p}=\int_{\mathbb{T}}|f(z)|^{p} \frac{1-|a|^{2}}{|1-\bar{a} z|^{2}} d \lambda \leq \frac{1+|a|}{1-|a|}\|f\|_{H^{p}}^{p} .
$$

Hence

$$
\left\|C_{q_{a}}\right\| \leq\left(\frac{1+|a|}{1-|a|}\right)^{1 / p}
$$




\section{Boundedness}

Let us treat the case of the special (but important) case of Moebius transformations, which are automorphisms of the disk. Consider the Moebius transformation $q_{a}(z)=\frac{a-z}{1-\bar{a} z} \quad$, where $a \in \mathbb{D}$.

For every polynomial $f$, we have

$$
\left\|f \circ q_{a}\right\|_{H^{p}}^{p}=\int_{\mathbb{T}}|f(z)|^{p} \frac{1-|a|^{2}}{|1-\bar{a} z|^{2}} d \lambda \leq \frac{1+|a|}{1-|a|}\|f\|_{H^{p}}^{p} .
$$

Actually

$$
\left\|C_{q_{a}}\right\|=\left(\frac{1+|a|}{1-|a|}\right)^{1 / p}
$$


We can now reduce the problem to $\varphi(0)=0$. Indeed, 
We can now reduce the problem to $\varphi(0)=0$. Indeed,

Write $a=\varphi(0) \quad$ and consider $\quad \phi=q_{a} \circ \varphi \Longleftrightarrow q_{a} \circ \phi=\varphi$ 
We can now reduce the problem to $\varphi(0)=0$. Indeed,

Write $a=\varphi(0) \quad$ and consider $\quad \phi=q_{a} \circ \varphi \Longleftrightarrow q_{a} \circ \phi=\varphi$ we have $\phi(0)=0$. 
We can now reduce the problem to $\varphi(0)=0$. Indeed,

Write $a=\varphi(0) \quad$ and consider $\quad \phi=q_{a} \circ \varphi \Longleftrightarrow q_{a} \circ \phi=\varphi$

$$
\text { we have } \phi(0)=0 \text {. }
$$

Hence if we prove that $C_{\phi}$ is bounded (with $\left\|C_{\phi}\right\|=1$ ):

And

$$
C_{\varphi}=C_{\phi} \circ C_{q_{a}} \text { is bounded as well ! }
$$


We can now reduce the problem to $\varphi(0)=0$. Indeed,

Write $a=\varphi(0) \quad$ and consider $\quad \phi=q_{a} \circ \varphi \Longleftrightarrow q_{a} \circ \phi=\varphi$

$$
\text { we have } \phi(0)=0 \text {. }
$$

Hence if we prove that $C_{\phi}$ is bounded (with $\left\|C_{\phi}\right\|=1$ ):

And

$$
C_{\varphi}=C_{\phi} \circ C_{q_{a}} \text { is bounded as well ! }
$$

$$
\left\|C_{\varphi}\right\| \leq\left(\frac{1+|\varphi(0)|}{1-|\varphi(0)|}\right)^{1 / p}
$$




\section{Boundedness via the subordination principle}

Let $\varphi: \mathbb{D} \rightarrow \mathbb{D}$ be an analytic function with $\varphi(0)=0$, and $g: \mathbb{D} \rightarrow[0,+\infty)$ a subharmonic function. We have for every $r \in(0,1)$

$$
\int_{0}^{2 \pi} g\left(\varphi\left(r e^{i t}\right)\right) d t \leq \int_{0}^{2 \pi} g\left(r e^{i t}\right) d t .
$$




\section{Boundedness via the subordination principle}

Let $\varphi: \mathbb{D} \rightarrow \mathbb{D}$ be an analytic function with $\varphi(0)=0$, and $g: \mathbb{D} \rightarrow[0,+\infty)$ a subharmonic function. We have for every $r \in(0,1)$

$$
\int_{0}^{2 \pi} g\left(\varphi\left(r e^{i t}\right)\right) d t \leq \int_{0}^{2 \pi} g\left(r e^{i t}\right) d t .
$$

Indeed,

let $G$ be an harmonic function such that $G=g$ on $r \mathbb{T}$ and $g \leq G$ on $r \mathbb{D}$. 


\section{Boundedness via the subordination principle}

Let $\varphi: \mathbb{D} \rightarrow \mathbb{D}$ be an analytic function with $\varphi(0)=0$, and $g: \mathbb{D} \rightarrow[0,+\infty)$ a subharmonic function. We have for every $r \in(0,1)$

$$
\int_{0}^{2 \pi} g\left(\varphi\left(r e^{i t}\right)\right) d t \leq \int_{0}^{2 \pi} g\left(r e^{i t}\right) d t .
$$

Indeed,

let $G$ be an harmonic function such that $G=g$ on $r \mathbb{T}$ and $g \leq G$ on $r \mathbb{D}$.

Think to the Schwarz lemma!

$$
\frac{1}{2 \pi} \int_{0}^{2 \pi} g\left(\varphi\left(r e^{i t}\right)\right) d t \leq \frac{1}{2 \pi} \int_{0}^{2 \pi} G\left(\varphi\left(r e^{i t}\right)\right) d t=
$$




\section{Boundedness via the subordination principle}

Let $\varphi: \mathbb{D} \rightarrow \mathbb{D}$ be an analytic function with $\varphi(0)=0$, and $g: \mathbb{D} \rightarrow[0,+\infty)$ a subharmonic function. We have for every $r \in(0,1)$

$$
\int_{0}^{2 \pi} g\left(\varphi\left(r e^{i t}\right)\right) d t \leq \int_{0}^{2 \pi} g\left(r e^{i t}\right) d t .
$$

Indeed,

let $G$ be an harmonic function such that $G=g$ on $r \mathbb{T}$ and $g \leq G$ on $r \mathbb{D}$.

Do not forget that $G \circ \varphi$ is harmonic!

$$
\frac{1}{2 \pi} \int_{0}^{2 \pi} g\left(\varphi\left(r e^{i t}\right)\right) d t \leq \frac{1}{2 \pi} \int_{0}^{2 \pi} G\left(\varphi\left(r e^{i t}\right)\right) d t=G \circ \varphi(0)=
$$




\section{Boundedness via the subordination principle}

Let $\varphi: \mathbb{D} \rightarrow \mathbb{D}$ be an analytic function with $\varphi(0)=0$, and $g: \mathbb{D} \rightarrow[0,+\infty)$ a subharmonic function. We have for every $r \in(0,1)$

$$
\int_{0}^{2 \pi} g\left(\varphi\left(r e^{i t}\right)\right) d t \leq \int_{0}^{2 \pi} g\left(r e^{i t}\right) d t .
$$

Indeed,

let $G$ be an harmonic function such that $G=g$ on $r \mathbb{T}$ and $g \leq G$ on $r \mathbb{D}$.

Do not forget that $G \circ \varphi$ is harmonic!

$$
\begin{aligned}
\frac{1}{2 \pi} \int_{0}^{2 \pi} g\left(\varphi\left(r e^{i t}\right)\right) d t & \leq \frac{1}{2 \pi} \int_{0}^{2 \pi} G\left(\varphi\left(r e^{i t}\right)\right) d t=G \circ \varphi(0)=G(0) \\
& \leq G(0)=
\end{aligned}
$$




\section{Boundedness via the subordination principle}

Let $\varphi: \mathbb{D} \rightarrow \mathbb{D}$ be an analytic function with $\varphi(0)=0$, and $g: \mathbb{D} \rightarrow[0,+\infty)$ a subharmonic function. We have for every $r \in(0,1)$

$$
\int_{0}^{2 \pi} g\left(\varphi\left(r e^{i t}\right)\right) d t \leq \int_{0}^{2 \pi} g\left(r e^{i t}\right) d t .
$$

Indeed,

let $G$ be an harmonic function such that $G=g$ on $r \mathbb{T}$ and $g \leq G$ on $r \mathbb{D}$.

Do not forget that $G \circ \varphi$ is harmonic!

$$
\begin{aligned}
\frac{1}{2 \pi} \int_{0}^{2 \pi} g\left(\varphi\left(r e^{i t}\right)\right) d t & \leq \frac{1}{2 \pi} \int_{0}^{2 \pi} G\left(\varphi\left(r e^{i t}\right)\right) d t=G \circ \varphi(0)=G(0) \\
& \leq G(0)=\frac{1}{2 \pi} \int_{0}^{2 \pi} G\left(r e^{i t}\right) d t=
\end{aligned}
$$




\section{Boundedness via the subordination principle}

Let $\varphi: \mathbb{D} \rightarrow \mathbb{D}$ be an analytic function with $\varphi(0)=0$, and $g: \mathbb{D} \rightarrow[0,+\infty)$ a subharmonic function. We have for every $r \in(0,1)$

$$
\int_{0}^{2 \pi} g\left(\varphi\left(r e^{i t}\right)\right) d t \leq \int_{0}^{2 \pi} g\left(r e^{i t}\right) d t .
$$

Indeed,

let $G$ be an harmonic function such that $G=g$ on $r \mathbb{T}$ and $g \leq G$ on $r \mathbb{D}$.

Do not forget that $G \circ \varphi$ is harmonic!

$$
\begin{aligned}
\frac{1}{2 \pi} \int_{0}^{2 \pi} g\left(\varphi\left(r e^{i t}\right)\right) d t & \leq \frac{1}{2 \pi} \int_{0}^{2 \pi} G\left(\varphi\left(r e^{i t}\right)\right) d t=G \circ \varphi(0)=G(0) \\
& \leq G(0)=\frac{1}{2 \pi} \int_{0}^{2 \pi} G\left(r e^{i t}\right) d t=\frac{1}{2 \pi} \int_{0}^{2 \pi} g\left(r e^{i t}\right) d t .
\end{aligned}
$$




\section{Boundedness via the subordination principle}

Let $\varphi: \mathbb{D} \rightarrow \mathbb{D}$ be an analytic function with $\varphi(0)=0$, and $g: \mathbb{D} \rightarrow[0,+\infty)$ a subharmonic function. We have for every $r \in(0,1)$

$$
\int_{0}^{2 \pi} g\left(\varphi\left(r e^{i t}\right)\right) d t \leq \int_{0}^{2 \pi} g\left(r e^{i t}\right) d t .
$$

Indeed,

let $G$ be an harmonic function such that $G=g$ on $r \mathbb{T}$ and $g \leq G$ on $r \mathbb{D}$.

$$
\begin{aligned}
\frac{1}{2 \pi} \int_{0}^{2 \pi} g\left(\varphi\left(r e^{i t}\right)\right) d t & \leq \frac{1}{2 \pi} \int_{0}^{2 \pi} G\left(\varphi\left(r e^{i t}\right)\right) d t=G \circ \varphi(0)=G(0) \\
& \leq G(0)=\frac{1}{2 \pi} \int_{0}^{2 \pi} G\left(r e^{i t}\right) d t=\frac{1}{2 \pi} \int_{0}^{2 \pi} g\left(r e^{i t}\right) d t .
\end{aligned}
$$

Now apply this to $g(z)=|f(z)|^{p}$, where $f \in H^{p}$. 


\section{Boundedness via the subordination principle}

Let $\varphi: \mathbb{D} \rightarrow \mathbb{D}$ be an analytic function with $\varphi(0)=0$, and $g: \mathbb{D} \rightarrow[0,+\infty)$ a subharmonic function. We have for every $r \in(0,1)$

$$
\int_{0}^{2 \pi} g\left(\varphi\left(r e^{i t}\right)\right) d t \leq \int_{0}^{2 \pi} g\left(r e^{i t}\right) d t .
$$

Indeed,

let $G$ be an harmonic function such that $G=g$ on $r \mathbb{T}$ and $g \leq G$ on $r \mathbb{D}$.

$$
\begin{aligned}
\frac{1}{2 \pi} \int_{0}^{2 \pi} g\left(\varphi\left(r e^{i t}\right)\right) d t & \leq \frac{1}{2 \pi} \int_{0}^{2 \pi} G\left(\varphi\left(r e^{i t}\right)\right) d t=G \circ \varphi(0)=G(0) \\
& \leq G(0)=\frac{1}{2 \pi} \int_{0}^{2 \pi} G\left(r e^{i t}\right) d t=\frac{1}{2 \pi} \int_{0}^{2 \pi} g\left(r e^{i t}\right) d t .
\end{aligned}
$$

Now apply this to $g(z)=|f(z)|^{p}$, where $f \in H^{p}$. Letting $r \nearrow 1^{-}$, we get 


\section{Boundedness via the subordination principle}

Let $\varphi: \mathbb{D} \rightarrow \mathbb{D}$ be an analytic function with $\varphi(0)=0$, and $g: \mathbb{D} \rightarrow[0,+\infty)$ a subharmonic function. We have for every $r \in(0,1)$

$$
\int_{0}^{2 \pi} g\left(\varphi\left(r e^{i t}\right)\right) d t \leq \int_{0}^{2 \pi} g\left(r e^{i t}\right) d t .
$$

Indeed,

let $G$ be an harmonic function such that $G=g$ on $r \mathbb{T}$ and $g \leq G$ on $r \mathbb{D}$.

$$
\begin{aligned}
\frac{1}{2 \pi} \int_{0}^{2 \pi} g\left(\varphi\left(r e^{i t}\right)\right) d t & \leq \frac{1}{2 \pi} \int_{0}^{2 \pi} G\left(\varphi\left(r e^{i t}\right)\right) d t=G \circ \varphi(0)=G(0) \\
& \leq G(0)=\frac{1}{2 \pi} \int_{0}^{2 \pi} G\left(r e^{i t}\right) d t=\frac{1}{2 \pi} \int_{0}^{2 \pi} g\left(r e^{i t}\right) d t .
\end{aligned}
$$

Now apply this to $g(z)=|f(z)|^{p}$, where $f \in H^{p}$. Letting $r \nearrow 1^{-}$, we get

$$
\|f \circ \varphi\|_{H^{p}}^{p} \leq\|f\|_{H^{p}}^{p} \quad \text { i.e. }
$$




\section{Boundedness via the subordination principle}

Let $\varphi: \mathbb{D} \rightarrow \mathbb{D}$ be an analytic function with $\varphi(0)=0$, and $g: \mathbb{D} \rightarrow[0,+\infty)$ a subharmonic function. We have for every $r \in(0,1)$

$$
\int_{0}^{2 \pi} g\left(\varphi\left(r e^{i t}\right)\right) d t \leq \int_{0}^{2 \pi} g\left(r e^{i t}\right) d t .
$$

Indeed,

let $G$ be an harmonic function such that $G=g$ on $r \mathbb{T}$ and $g \leq G$ on $r \mathbb{D}$.

$$
\begin{aligned}
\frac{1}{2 \pi} \int_{0}^{2 \pi} g\left(\varphi\left(r e^{i t}\right)\right) d t & \leq \frac{1}{2 \pi} \int_{0}^{2 \pi} G\left(\varphi\left(r e^{i t}\right)\right) d t=G \circ \varphi(0)=G(0) \\
& \leq G(0)=\frac{1}{2 \pi} \int_{0}^{2 \pi} G\left(r e^{i t}\right) d t=\frac{1}{2 \pi} \int_{0}^{2 \pi} g\left(r e^{i t}\right) d t .
\end{aligned}
$$

Now apply this to $g(z)=|f(z)|^{p}$, where $f \in H^{p}$. Letting $r \nearrow 1^{-}$, we get

$$
\|f \circ \varphi\|_{H^{p}}^{p} \leq\|f\|_{H^{p}}^{p} \quad \text { i.e. } \quad\left\|C_{\varphi}\right\|=1
$$

The boundedness is proved!!

\section{Curso Internacional de Análisis Matemático en Andalucía}




\section{Boundedness via the Nevanlinna function}

$$
N_{\varphi}(w)=\left\{\begin{array}{cc}
\sum_{\varphi(\alpha)=w} \log \frac{1}{|\alpha|} \quad \text { if } w \neq \varphi(0) \text { and } w \in \varphi(\mathbb{D}) \\
0 \quad \begin{array}{l}
\text { else. } \\
\text { (every } \alpha \text { occurs as many times as its multiplicity) }
\end{array}
\end{array}\right.
$$




\section{Boundedness via the Nevanlinna function}

$$
N_{\varphi}(w)=\left\{\begin{array}{cl}
\sum_{\varphi(\alpha)=w} \log \frac{1}{|\alpha|} & \text { if } w \neq \varphi(0) \text { and } w \in \varphi(\mathbb{D}) \\
0 & \text { else. }
\end{array}\right.
$$

(every $\alpha$ occurs as many times as its multiplicity)

\section{(Littlewood)}

$\forall w \neq \varphi(0), \quad N_{\varphi}(w) \leq \log \left|\frac{1-\overline{\varphi(0)} w}{\varphi(0)-w}\right| \quad=O((1-|w|)) \quad$ when $|w| \rightarrow 1^{-}$. 


\section{Boundedness via the Nevanlinna function}

$$
N_{\varphi}(w)=\left\{\begin{array}{cl}
\sum_{\varphi(\alpha)=w} \log \frac{1}{|\alpha|} & \text { if } w \neq \varphi(0) \text { and } w \in \varphi(\mathbb{D}) \\
0 & \text { else. }
\end{array}\right.
$$

(every $\alpha$ occurs as many times as its multiplicity)

\section{(Littlewood)}

$\forall w \neq \varphi(0), \quad N_{\varphi}(w) \leq \log \left|\frac{1-\overline{\varphi(0)} w}{\varphi(0)-w}\right| \quad=O((1-|w|)) \quad$ when $|w| \rightarrow 1^{-}$.

This very nice inequality is a "super Schwarz" lemma: it means, when $\varphi(0)=0$ 


\section{Boundedness via the Nevanlinna function}

$$
N_{\varphi}(w)=\left\{\begin{array}{cl}
\sum_{\varphi(\alpha)=w} \log \frac{1}{|\alpha|} & \text { if } w \neq \varphi(0) \text { and } w \in \varphi(\mathbb{D}) \\
0 & \text { else. }
\end{array}\right.
$$

(every $\alpha$ occurs as many times as its multiplicity)

\section{(Littlewood)}

$\forall w \neq \varphi(0), \quad N_{\varphi}(w) \leq \log \left|\frac{1-\overline{\varphi(0)} w}{\varphi(0)-w}\right| \quad=O((1-|w|)) \quad$ when $|w| \rightarrow 1^{-}$.

This very nice inequality is a "super Schwarz" lemma: it means, when $\varphi(0)=0$

$$
|\varphi(z)| \leq \prod_{\varphi(\alpha)=\varphi(z)}|\alpha|
$$




\section{Boundedness via the Nevanlinna function}

$$
N_{\varphi}(w)=\left\{\begin{array}{cl}
\sum_{\varphi(\alpha)=w} \log \frac{1}{|\alpha|} & \text { if } w \neq \varphi(0) \text { and } w \in \varphi(\mathbb{D}) \\
0 & \text { else. }
\end{array}\right.
$$

(every $\alpha$ occurs as many times as its multiplicity)

\section{(Littlewood)}

$\forall w \neq \varphi(0), \quad N_{\varphi}(w) \leq \log \left|\frac{1-\overline{\varphi(0)} w}{\varphi(0)-w}\right| \quad=O((1-|w|)) \quad$ when $|w| \rightarrow 1^{-}$.

This very nice inequality is a "super Schwarz" lemma: it means, when $\varphi(0)=0$

$$
|\varphi(z)| \leq \prod_{\varphi(\alpha)=\varphi(z)}|\alpha| \quad(\leq|z|) .
$$




\section{Boundedness via the Nevanlinna function}

$$
N_{\varphi}(w)=\left\{\begin{array}{cl}
\sum_{\varphi(\alpha)=w} \log \frac{1}{|\alpha|} & \text { if } w \neq \varphi(0) \text { and } w \in \varphi(\mathbb{D}) \\
0 & \text { else. }
\end{array}\right.
$$

(every $\alpha$ occurs as many times as its multiplicity)

\section{(Littlewood)}

$\forall w \neq \varphi(0), \quad N_{\varphi}(w) \leq \log \left|\frac{1-\overline{\varphi(0)} w}{\varphi(0)-w}\right| \quad=O((1-|w|)) \quad$ when $|w| \rightarrow 1^{-}$.

This very nice inequality is a "super Schwarz" lemma: it means, when $\varphi(0)=0$

$$
|\varphi(z)| \leq \prod_{\varphi(\alpha)=\varphi(z)}|\alpha| \quad(\leq|z|) .
$$

Now, the Littewood-Paley formula $\underline{(p=2)}$ 


\section{Boundedness via the Nevanlinna function}

$$
N_{\varphi}(w)=\left\{\begin{array}{cl}
\sum_{\varphi(\alpha)=w} \log \frac{1}{|\alpha|} & \text { if } w \neq \varphi(0) \text { and } w \in \varphi(\mathbb{D}) \\
0 & \text { else. }
\end{array}\right.
$$

(every $\alpha$ occurs as many times as its multiplicity)

\section{(Littlewood)}

$\forall w \neq \varphi(0), \quad N_{\varphi}(w) \leq \log \left|\frac{1-\overline{\varphi(0)} w}{\varphi(0)-w}\right| \quad=O((1-|w|)) \quad$ when $|w| \rightarrow 1^{-}$.

This very nice inequality is a "super Schwarz" lemma: it means, when $\varphi(0)=0$

$$
|\varphi(z)| \leq \prod_{\varphi(\alpha)=\varphi(z)}|\alpha| \quad(\leq|z|) .
$$

Now, the Littewood-Paley formula $\underline{(p=2)}$

$$
\|f\|_{2}^{2}=|f(0)|^{2}+2 \int_{\mathbb{D}}\left|f^{\prime}\right|^{2} \log \frac{1}{|z|} d \mathcal{A}
$$

implies again the boundedness of $C_{\varphi}$ is bounded on $H^{2}$.

\section{Curso Internacional de Análisis Matemático en Andalucía}




\section{Boundedness via the Nevanlinna function}

Indeed:

$$
\|f \circ \varphi\|_{2}^{2}=|f \circ \varphi(0)|^{2}+2 \int_{\mathbb{D}}\left|(f \circ \varphi)^{\prime}\right|^{2} \log \frac{1}{|z|} d \mathcal{A}
$$




\section{Boundedness via the Nevanlinna function}

Indeed:

$$
\begin{aligned}
\|f \circ \varphi\|_{2}^{2} & =|f \circ \varphi(0)|^{2}+2 \int_{\mathbb{D}}\left|(f \circ \varphi)^{\prime}\right|^{2} \log \frac{1}{|z|} d \mathcal{A} \\
& =|f \circ \varphi(0)|^{2}+2 \int_{\mathbb{D}}\left(\left|f^{\prime}\right|^{2} \circ \varphi\right) \times\left|\varphi^{\prime}\right|^{2} \log \frac{1}{|z|} d \mathcal{A}
\end{aligned}
$$




\section{Boundedness via the Nevanlinna function}

Indeed:

$$
\begin{aligned}
\|f \circ \varphi\|_{2}^{2} & =|f \circ \varphi(0)|^{2}+2 \int_{\mathbb{D}}\left|(f \circ \varphi)^{\prime}\right|^{2} \log \frac{1}{|z|} d \mathcal{A} \\
& =|f \circ \varphi(0)|^{2}+2 \int_{\mathbb{D}}\left(\left|f^{\prime}\right|^{2} \circ \varphi\right) \times\left|\varphi^{\prime}\right|^{2} \log \frac{1}{|z|} d \mathcal{A} \\
& =|f \circ \varphi(0)|^{2}+2 \int_{\mathbb{D}}\left|f^{\prime}\right|^{2} N_{\varphi} d \mathcal{A}
\end{aligned}
$$




\section{Boundedness via the Nevanlinna function}

Indeed:

$$
\begin{aligned}
\|f \circ \varphi\|_{2}^{2} & =|f \circ \varphi(0)|^{2}+2 \int_{\mathbb{D}}\left|(f \circ \varphi)^{\prime}\right|^{2} \log \frac{1}{|z|} d \mathcal{A} \\
& =|f \circ \varphi(0)|^{2}+2 \int_{\mathbb{D}}\left(\left|f^{\prime}\right|^{2} \circ \varphi\right) \times\left|\varphi^{\prime}\right|^{2} \log \frac{1}{|z|} d \mathcal{A} \\
& =|f \circ \varphi(0)|^{2}+2 \int_{\mathbb{D}}\left|f^{\prime}\right|^{2} N_{\varphi} d \mathcal{A}
\end{aligned}
$$

Now, in the case $\varphi(0)=0$, using the majorization $N_{\varphi}(w) \leq \log \left|\frac{1}{w}\right|$, we get 


\section{Boundedness via the Nevanlinna function}

Indeed:

$$
\begin{aligned}
\|f \circ \varphi\|_{2}^{2} & =|f \circ \varphi(0)|^{2}+2 \int_{\mathbb{D}}\left|(f \circ \varphi)^{\prime}\right|^{2} \log \frac{1}{|z|} d \mathcal{A} \\
& =|f \circ \varphi(0)|^{2}+2 \int_{\mathbb{D}}\left(\left|f^{\prime}\right|^{2} \circ \varphi\right) \times\left|\varphi^{\prime}\right|^{2} \log \frac{1}{|z|} d \mathcal{A} \\
& =|f \circ \varphi(0)|^{2}+2 \int_{\mathbb{D}}\left|f^{\prime}\right|^{2} N_{\varphi} d \mathcal{A}
\end{aligned}
$$

Now, in the case $\varphi(0)=0$, using the majorization $N_{\varphi}(w) \leq \log \left|\frac{1}{w}\right|$, we get

$$
\|f \circ \varphi\|_{2}^{2} \leq|f(0)|^{2}+2 \int_{\mathbb{D}}\left|f^{\prime}\right|^{2} \log \left|\frac{1}{w}\right| d \mathcal{A}
$$




\section{Boundedness via the Nevanlinna function}

Indeed:

$$
\begin{aligned}
\|f \circ \varphi\|_{2}^{2} & =|f \circ \varphi(0)|^{2}+2 \int_{\mathbb{D}}\left|(f \circ \varphi)^{\prime}\right|^{2} \log \frac{1}{|z|} d \mathcal{A} \\
& =|f \circ \varphi(0)|^{2}+2 \int_{\mathbb{D}}\left(\left|f^{\prime}\right|^{2} \circ \varphi\right) \times\left|\varphi^{\prime}\right|^{2} \log \frac{1}{|z|} d \mathcal{A} \\
& =|f \circ \varphi(0)|^{2}+2 \int_{\mathbb{D}}\left|f^{\prime}\right|^{2} N_{\varphi} d \mathcal{A}
\end{aligned}
$$

Now, in the case $\varphi(0)=0$, using the majorization $N_{\varphi}(w) \leq \log \left|\frac{1}{w}\right|$, we get

$$
\|f \circ \varphi\|_{2}^{2} \leq|f(0)|^{2}+2 \int_{\mathbb{D}}\left|f^{\prime}\right|^{2} \log \left|\frac{1}{W}\right| d \mathcal{A}=\|f\|_{2}^{2}
$$

and

$$
\left\|C_{\varphi}\right\| \leq 1
$$




\section{Boundedness from the Carleson embedding point of view}

Point out that

$$
\|f \circ \varphi\|_{p}^{p}=\int_{\mathbb{D}}|f|^{p} d \lambda_{\varphi} \quad \text { with } \lambda_{\varphi}(E)=\lambda\left(\varphi^{*-1}(E)\right)
$$

where $E \subset \overline{\mathbb{D}}$ (Borel). 


\section{Boundedness from the Carleson embedding point of view}

Point out that

$$
\|f \circ \varphi\|_{p}^{p}=\int_{\mathbb{D}}|f|^{p} d \lambda_{\varphi} \quad \text { with } \lambda_{\varphi}(E)=\lambda\left(\varphi^{*-1}(E)\right)
$$

where $E \subset \overline{\mathbb{D}}$ (Borel). The measure $\lambda_{\varphi}$ is the pullback measure associated to $\varphi$. 


\section{Boundedness from the Carleson embedding point of view}

Point out that

$$
\|f \circ \varphi\|_{p}^{p}=\int_{\overline{\mathbb{D}}}|f|^{p} d \lambda_{\varphi} \quad \text { with } \lambda_{\varphi}(E)=\lambda\left(\varphi^{*-1}(E)\right)
$$

where $E \subset \overline{\mathbb{D}}$ (Borel). The measure $\lambda_{\varphi}$ is the pullback measure associated to $\varphi$.

The boundedness of $C_{\varphi}$ on $H^{p}$ is equivalent to the boundedness of

$$
f \in H^{p} \longmapsto f \in L^{p}\left(\overline{\mathbb{D}}, \lambda_{\varphi}\right)
$$




\section{Boundedness from the Carleson embedding point of view}

Point out that

$$
\|f \circ \varphi\|_{p}^{p}=\int_{\mathbb{D}}|f|^{p} d \lambda_{\varphi} \quad \text { with } \lambda_{\varphi}(E)=\lambda\left(\varphi^{*-1}(E)\right)
$$

where $E \subset \overline{\mathbb{D}}$ (Borel). The measure $\lambda_{\varphi}$ is the pullback measure associated to $\varphi$.

The boundedness of $C_{\varphi}$ on $H^{p}$ is equivalent to the boundedness of

$$
f \in H^{p} \longmapsto f \in L^{p}\left(\overline{\mathbb{D}}, \lambda_{\varphi}\right)
$$

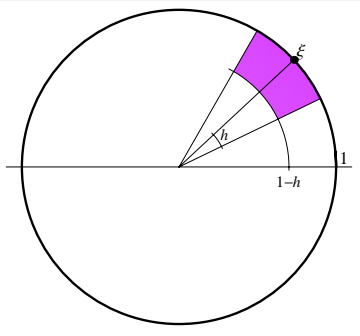

Thanks to the Carleson embedding theorem, it means that we control the size of the Carleson window $\mathcal{W}(\xi, h)$ 


\section{Boundedness from the Carleson embedding point of view}

Point out that

$$
\|f \circ \varphi\|_{p}^{p}=\int_{\overline{\mathbb{D}}}|f|^{p} d \lambda_{\varphi} \quad \text { with } \lambda_{\varphi}(E)=\lambda\left(\varphi^{*-1}(E)\right)
$$

where $E \subset \overline{\mathbb{D}}$ (Borel). The measure $\lambda_{\varphi}$ is the pullback measure associated to $\varphi$.

The boundedness of $C_{\varphi}$ on $H^{p}$ is equivalent to the boundedness of

$$
f \in H^{p} \longmapsto f \in L^{p}\left(\overline{\mathbb{D}}, \lambda_{\varphi}\right)
$$

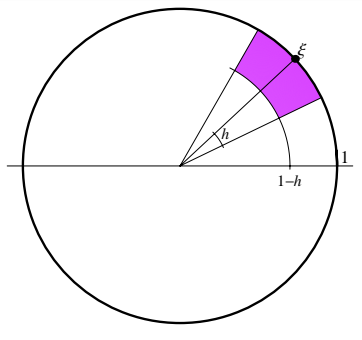

Thanks to the Carleson embedding theorem, it means that we control the size of the Carleson window $\mathcal{W}(\xi, h)$

$$
\rho_{\varphi}(h)=\sup _{\xi \in \mathbb{T}} \lambda_{\varphi}(\mathcal{W}(\xi, h))=O(h) \quad \text { when } h \rightarrow 0
$$




\section{Compactness}

\section{Definition}

An operator $T: X \rightarrow Y$ is compact if $T\left(B_{X}\right)$ is relatively compact in $Y$. 


\section{Compactness}

\section{Definition}

An operator $T: X \rightarrow Y$ is compact if $T\left(B_{X}\right)$ is relatively compact in $Y$.

First remarks (H.Schwartz,'68)

(1) The operator $C_{\varphi}: H^{p} \rightarrow H^{p}$ is compact if and only if for every bounded sequence $\left\{f_{n}\right\}_{n}$ in $H^{p}$ converging to 0 uniformly on compact subsets of $\mathbb{D}$, we have $f_{n} \circ \varphi \rightarrow 0$ in $H^{p}$. 


\section{Compactness}

\section{Definition}

An operator $T: X \rightarrow Y$ is compact if $T\left(B_{X}\right)$ is relatively compact in $Y$.

First remarks (H.Schwartz,'68)

(1) The operator $C_{\varphi}: H^{p} \rightarrow H^{p}$ is compact if and only if for every bounded sequence $\left\{f_{n}\right\}_{n}$ in $H^{p}$ converging to 0 uniformly on compact subsets of $\mathbb{D}$, we have $f_{n} \circ \varphi \rightarrow 0$ in $H^{p}$.

(2) If $C_{\varphi}$ is compact on $H^{p}$, then $\lambda_{\varphi}(\mathbb{T})=0$, i.e. $\left|\varphi^{*}\right|<1$ a.e.. 


\section{Compactness}

\section{Definition}

An operator $T: X \rightarrow Y$ is compact if $T\left(B_{X}\right)$ is relatively compact in $Y$.

First remarks (H.Schwartz,'68)

(1) The operator $C_{\varphi}: H^{p} \rightarrow H^{p}$ is compact if and only if for every bounded sequence $\left\{f_{n}\right\}_{n}$ in $H^{P}$ converging to 0 uniformly on compact subsets of $\mathbb{D}$, we have $f_{n} \circ \varphi \rightarrow 0$ in $H^{p}$.

(2) If $C_{\varphi}$ is compact on $H^{p}$, then $\lambda_{\varphi}(\mathbb{T})=0$, i.e. $\left|\varphi^{*}\right|<1$ a.e..

(3) If $C_{\varphi}$ is compact on $H^{p}$, then $\lim _{|z| \rightarrow 1^{-}} \frac{1-|z|}{1-|\varphi(z)|}=0$ 


\section{Compactness}

\section{Definition}

An operator $T: X \rightarrow Y$ is compact if $T\left(B_{X}\right)$ is relatively compact in $Y$.

First remarks (H.Schwartz,'68)

(1) The operator $C_{\varphi}: H^{p} \rightarrow H^{p}$ is compact if and only if for every bounded sequence $\left\{f_{n}\right\}_{n}$ in $H^{P}$ converging to 0 uniformly on compact subsets of $\mathbb{D}$, we have $f_{n} \circ \varphi \rightarrow 0$ in $H^{p}$.

(2) If $C_{\varphi}$ is compact on $H^{p}$, then $\lambda_{\varphi}(\mathbb{T})=0$, i.e. $\left|\varphi^{*}\right|<1$ a.e..

(3) If $C_{\varphi}$ is compact on $H^{p}$, then $\lim _{|z| \rightarrow 1^{-}} \frac{1-|z|}{1-|\varphi(z)|}=0$

Indeed

(1) Exercice with the help of Montel's theorem 


\section{Compactness}

\section{Definition}

An operator $T: X \rightarrow Y$ is compact if $T\left(B_{X}\right)$ is relatively compact in $Y$.

First remarks (H.Schwartz,'68)

(1) The operator $C_{\varphi}: H^{p} \rightarrow H^{p}$ is compact if and only if for every bounded sequence $\left\{f_{n}\right\}_{n}$ in $H^{P}$ converging to 0 uniformly on compact subsets of $\mathbb{D}$, we have $f_{n} \circ \varphi \rightarrow 0$ in $H^{p}$.

(2) If $C_{\varphi}$ is compact on $H^{p}$, then $\lambda_{\varphi}(\mathbb{T})=0$, i.e. $\left|\varphi^{*}\right|<1$ a.e..

(3) If $C_{\varphi}$ is compact on $H^{p}$, then $\lim _{|z| \rightarrow 1^{-}} \frac{1-|z|}{1-|\varphi(z)|}=0$

Indeed

(1) Exercice with the help of Montel's theorem (or use weak-star compactness). 


\section{Compactness}

\section{Definition}

An operator $T: X \rightarrow Y$ is compact if $T\left(B_{X}\right)$ is relatively compact in $Y$.

First remarks (H.Schwartz,'68)

(1) The operator $C_{\varphi}: H^{p} \rightarrow H^{p}$ is compact if and only if for every bounded sequence $\left\{f_{n}\right\}_{n}$ in $H^{p}$ converging to 0 uniformly on compact subsets of $\mathbb{D}$, we have $f_{n} \circ \varphi \rightarrow 0$ in $H^{p}$.

(2) If $C_{\varphi}$ is compact on $H^{p}$, then $\lambda_{\varphi}(\mathbb{T})=0$, i.e. $\left|\varphi^{*}\right|<1$ a.e..

(3) If $C_{\varphi}$ is compact on $H^{p}$, then $\lim _{|z| \rightarrow 1^{-}} \frac{1-|z|}{1-|\varphi(z)|}=0$

Indeed

(1) Exercice with the help of Montel's theorem (or use weak-star compactness).

(2) The sequence $\left(z^{n}\right)$ uniformly converges to 0 on compact subsets of $\mathbb{D}$, so

$$
\left\|C_{\varphi}\left(z^{n}\right)\right\|_{p}^{p}=\left\|\varphi^{n}\right\|_{p}^{p} \longrightarrow 0
$$

but 


\section{Compactness}

\section{Definition}

An operator $T: X \rightarrow Y$ is compact if $T\left(B_{X}\right)$ is relatively compact in $Y$.

First remarks (H.Schwartz,'68)

(1) The operator $C_{\varphi}: H^{p} \rightarrow H^{p}$ is compact if and only if for every bounded sequence $\left\{f_{n}\right\}_{n}$ in $H^{p}$ converging to 0 uniformly on compact subsets of $\mathbb{D}$, we have $f_{n} \circ \varphi \rightarrow 0$ in $H^{p}$.

(2) If $C_{\varphi}$ is compact on $H^{p}$, then $\lambda_{\varphi}(\mathbb{T})=0$, i.e. $\left|\varphi^{*}\right|<1$ a.e..

(3) If $C_{\varphi}$ is compact on $H^{p}$, then $\lim _{|z| \rightarrow 1^{-}} \frac{1-|z|}{1-|\varphi(z)|}=0$

Indeed

(1) Exercice with the help of Montel's theorem (or use weak-star compactness).

(2) The sequence $\left(z^{n}\right)$ uniformly converges to 0 on compact subsets of $\mathbb{D}$, so

$$
\left\|C_{\varphi}\left(z^{n}\right)\right\|_{p}^{p}=\left\|\varphi^{n}\right\|_{p}^{p} \longrightarrow 0
$$

but

$$
\left\|\varphi^{n}\right\|_{p}^{p}=\int_{\mathbb{T}}\left|\varphi^{*}\right|^{n p} d \lambda \longrightarrow \lambda_{\varphi}(\mathbb{T}) .
$$




\section{Compactness}

(3) If $C_{\varphi}$ is compact on $H^{p}$, then $\lim _{|z| \rightarrow 1^{-}} \frac{1-|z|}{1-|\varphi(z)|}=0$

Indeed 


\section{Compactness}

(3) If $C_{\varphi}$ is compact on $H^{p}$, then $\lim _{|z| \rightarrow 1^{-}} \frac{1-|z|}{1-|\varphi(z)|}=0$

Indeed

Remember that the functional $\delta_{z}$ has norm $\frac{1}{\left(1-|z|^{2}\right)^{1 / p}}$ and point out that $C_{\varphi}^{*}\left(\delta_{z}\right)=\delta_{\varphi(z)}$ 


\section{Compactness}

(3) If $C_{\varphi}$ is compact on $H^{p}$, then $\lim _{|z| \rightarrow 1^{-}} \frac{1-|z|}{1-|\varphi(z)|}=0$

Indeed

Remember that the functional $\delta_{z}$ has norm $\frac{1}{\left(1-|z|^{2}\right)^{1 / p}}$ and point out that $C_{\varphi}^{*}\left(\delta_{z}\right)=\delta_{\varphi(z)}$.

Then for any sequence $z_{n} \in \mathbb{D}$ such that $\left|z_{n}\right| \longrightarrow 1^{-}$, the sequence $\mu_{n}=\left(1-\left|z_{n}\right|^{2}\right)^{1 / p} \delta_{z_{n}}$ lies in the unit sphere of the dual of $H^{p}$. 


\section{Compactness}

(3) If $C_{\varphi}$ is compact on $H^{p}$, then $\lim _{|z| \rightarrow 1^{-}} \frac{1-|z|}{1-|\varphi(z)|}=0$

Indeed

Remember that the functional $\delta_{z}$ has norm $\frac{1}{\left(1-|z|^{2}\right)^{1 / p}}$ and point out that $C_{\varphi}^{*}\left(\delta_{z}\right)=\delta_{\varphi(z)}$.

Then for any sequence $z_{n} \in \mathbb{D}$ such that $\left|z_{n}\right| \longrightarrow 1^{-}$, the sequence $\mu_{n}=\left(1-\left|z_{n}\right|^{2}\right)^{1 / p} \delta_{z_{n}}$ lies in the unit sphere of the dual of $H^{p}$.

Since $C_{\varphi}^{*}$ is compact on $\left(H^{p}\right)^{*}$ and $\mu_{n}$ is weakstar convergent to 0 , 


\section{Compactness}

(3) If $C_{\varphi}$ is compact on $H^{p}$, then $\lim _{|z| \rightarrow 1^{-}} \frac{1-|z|}{1-|\varphi(z)|}=0$

Indeed

Remember that the functional $\delta_{z}$ has norm $\frac{1}{\left(1-|z|^{2}\right)^{1 / p}}$ and point out that $C_{\varphi}^{*}\left(\delta_{z}\right)=\delta_{\varphi(z)}$.

Then for any sequence $z_{n} \in \mathbb{D}$ such that $\left|z_{n}\right| \longrightarrow 1^{-}$, the sequence $\mu_{n}=\left(1-\left|z_{n}\right|^{2}\right)^{1 / p} \delta_{z_{n}}$ lies in the unit sphere of the dual of $H^{p}$.

Since $C_{\varphi}^{*}$ is compact on $\left(H^{p}\right)^{*}$ and $\mu_{n}$ is weakstar convergent to 0 , we have

$$
\left\|C_{\varphi}^{*}\left(\mu_{n}\right)\right\|_{\left(H^{p}\right)^{*}} \longrightarrow 0
$$

but 


\section{Compactness}

(3) If $C_{\varphi}$ is compact on $H^{p}$, then $\lim _{|z| \rightarrow 1^{-}} \frac{1-|z|}{1-|\varphi(z)|}=0$

Indeed

Remember that the functional $\delta_{z}$ has norm $\frac{1}{\left(1-|z|^{2}\right)^{1 / p}}$ and point out that $C_{\varphi}^{*}\left(\delta_{z}\right)=\delta_{\varphi(z)}$.

Then for any sequence $z_{n} \in \mathbb{D}$ such that $\left|z_{n}\right| \longrightarrow 1^{-}$, the sequence $\mu_{n}=\left(1-\left|z_{n}\right|^{2}\right)^{1 / p} \delta_{z_{n}}$ lies in the unit sphere of the dual of $H^{p}$.

Since $C_{\varphi}^{*}$ is compact on $\left(H^{p}\right)^{*}$ and $\mu_{n}$ is weakstar convergent to 0 , we have

$$
\left\|C_{\varphi}^{*}\left(\mu_{n}\right)\right\|_{\left(H^{p}\right)^{*}} \longrightarrow 0
$$

but

$$
\left\|C_{\varphi}^{*}\left(\mu_{n}\right)\right\|_{\left(H^{p}\right)^{*}}=\left(1-\left|z_{n}\right|^{2}\right)^{1 / p}\left\|\delta_{\varphi\left(z_{n}\right)}\right\|_{\left(H^{p}\right)^{*}}=
$$




\section{Compactness}

(3) If $C_{\varphi}$ is compact on $H^{p}$, then $\lim _{|z| \rightarrow 1^{-}} \frac{1-|z|}{1-|\varphi(z)|}=0$

Indeed

Remember that the functional $\delta_{z}$ has norm $\frac{1}{\left(1-|z|^{2}\right)^{1 / p}}$ and point out that $C_{\varphi}^{*}\left(\delta_{z}\right)=\delta_{\varphi(z)}$.

Then for any sequence $z_{n} \in \mathbb{D}$ such that $\left|z_{n}\right| \longrightarrow 1^{-}$, the sequence $\mu_{n}=\left(1-\left|z_{n}\right|^{2}\right)^{1 / p} \delta_{z_{n}}$ lies in the unit sphere of the dual of $H^{p}$.

Since $C_{\varphi}^{*}$ is compact on $\left(H^{p}\right)^{*}$ and $\mu_{n}$ is weakstar convergent to 0 , we have

$$
\left\|C_{\varphi}^{*}\left(\mu_{n}\right)\right\|_{\left(H^{p}\right)^{*}} \longrightarrow 0
$$

but

$$
\left\|C_{\varphi}^{*}\left(\mu_{n}\right)\right\|_{\left(H^{p}\right)^{*}}=\left(1-\left|z_{n}\right|^{2}\right)^{1 / p}\left\|\delta_{\varphi\left(z_{n}\right)}\right\|_{\left(H^{p}\right)^{*}}=\frac{\left(1-\left|z_{n}\right|^{2}\right)^{1 / p}}{\left(1-\left|\varphi\left(z_{n}\right)\right|^{2}\right)^{1 / p}}
$$




\section{Compactness}

\section{(Shapiro-Taylor '73) The problem reduces to the hilbertian case:}
$C_{\varphi}$ is compact on $H^{p}$ if and only if $C_{\varphi}$ is compact on $H^{2}$. 


\section{Compactness}

\section{(Shapiro-Taylor '73) The problem reduces to the hilbertian case:}
$C_{\varphi}$ is compact on $H^{p}$ if and only if $C_{\varphi}$ is compact on $H^{2}$.

If $p, q \geq 1$ and $C_{\varphi}$ is compact on $H^{q}$ then $C_{\varphi}$ is compact on $H^{p}$. Indeed, 


\section{Compactness}

\section{(Shapiro-Taylor '73) The problem reduces to the hilbertian case:}
$C_{\varphi}$ is compact on $H^{p}$ if and only if $C_{\varphi}$ is compact on $H^{2}$.

If $p, q \geq 1$ and $C_{\varphi}$ is compact on $H^{q}$ then $C_{\varphi}$ is compact on $H^{p}$. Indeed, Take $\left\{f_{n}\right\}_{n} \in B_{H^{p}}$ uniformly converging to 0 on compact subsets of $\mathbb{D}$. 


\section{Compactness}

\section{(Shapiro-Taylor '73) The problem reduces to the hilbertian case:}

$\mathrm{C}_{\varphi}$ is compact on $\mathrm{H}^{p} \quad$ if and only if $\mathrm{C}_{\varphi}$ is compact on $\mathrm{H}^{2}$.

If $p, q \geq 1$ and $C_{\varphi}$ is compact on $H^{q}$ then $C_{\varphi}$ is compact on $H^{p}$. Indeed,

Take $\left\{f_{n}\right\}_{n} \in B_{H^{p}}$ uniformly converging to 0 on compact subsets of $\mathbb{D}$.

Write $f_{n}=B_{n} \cdot g_{n}$ with $\left|B_{n}^{*}\right|=1$ a.e. and $g_{n} \in H^{p}$ without zeros in $\mathbb{D}$. 


\section{Compactness}

\section{(Shapiro-Taylor '73) The problem reduces to the hilbertian case:}

$\mathrm{C}_{\varphi}$ is compact on $\mathrm{H}^{p} \quad$ if and only if $\mathrm{C}_{\varphi}$ is compact on $\mathrm{H}^{2}$.

If $p, q \geq 1$ and $C_{\varphi}$ is compact on $H^{q}$ then $C_{\varphi}$ is compact on $H^{p}$. Indeed,

Take $\left\{f_{n}\right\}_{n} \in B_{H^{p}}$ uniformly converging to 0 on compact subsets of $\mathbb{D}$.

Write $f_{n}=B_{n} \cdot g_{n}$ with $\left|B_{n}^{*}\right|=1$ a.e. and $g_{n} \in H^{p}$ without zeros in $\mathbb{D}$.

The sequence $G_{n}=g_{n}^{p / q}$ is defined and lies in $B_{H^{q}}$. 


\section{Compactness}

\section{(Shapiro-Taylor '73) The problem reduces to the hilbertian case:}

$\mathrm{C}_{\varphi}$ is compact on $\mathrm{H}^{p} \quad$ if and only if $\mathrm{C}_{\varphi}$ is compact on $\mathrm{H}^{2}$.

If $p, q \geq 1$ and $C_{\varphi}$ is compact on $H^{q}$ then $C_{\varphi}$ is compact on $H^{p}$. Indeed,

Take $\left\{f_{n}\right\}_{n} \in B_{H^{p}}$ uniformly converging to 0 on compact subsets of $\mathbb{D}$.

Write $f_{n}=B_{n} \cdot g_{n}$ with $\left|B_{n}^{*}\right|=1$ a.e. and $g_{n} \in H^{p}$ without zeros in $\mathbb{D}$.

The sequence $G_{n}=g_{n}^{p / q}$ is defined and lies in $B_{H^{q}}$.

Up to (enough) subsequences, we may assume that

$G_{n} \longrightarrow G \in B_{H^{q}}, \quad B_{n} \longrightarrow B \in B_{H^{\infty}} \quad$ uniformly on compact subsets of $\mathbb{D}$ and 


\section{Compactness}

\section{(Shapiro-Taylor '73) The problem reduces to the hilbertian case:}

$C_{\varphi}$ is compact on $H^{p} \quad$ if and only if $C_{\varphi}$ is compact on $H^{2}$.

If $p, q \geq 1$ and $C_{\varphi}$ is compact on $H^{q}$ then $C_{\varphi}$ is compact on $H^{p}$. Indeed,

Take $\left\{f_{n}\right\}_{n} \in B_{H^{p}}$ uniformly converging to 0 on compact subsets of $\mathbb{D}$.

Write $f_{n}=B_{n} \cdot g_{n}$ with $\left|B_{n}^{*}\right|=1$ a.e. and $g_{n} \in H^{p}$ without zeros in $\mathbb{D}$.

The sequence $G_{n}=g_{n}^{p / q}$ is defined and lies in $B_{H^{q}}$.

Up to (enough) subsequences, we may assume that

$G_{n} \longrightarrow G \in B_{H^{q}}, \quad B_{n} \longrightarrow B \in B_{H^{\infty}} \quad$ uniformly on compact subsets of $\mathbb{D}$ and the sequence $\left(G_{n} \circ \varphi\right)$ converges to $G \circ \varphi$ in $H^{q}$ (since $C_{\varphi} \in \mathcal{K}\left(H^{q}\right)$ ) 


\section{Compactness}

\section{(Shapiro-Taylor '73) The problem reduces to the hilbertian case:}

$C_{\varphi}$ is compact on $H^{p} \quad$ if and only if $C_{\varphi}$ is compact on $H^{2}$.

If $p, q \geq 1$ and $C_{\varphi}$ is compact on $H^{q}$ then $C_{\varphi}$ is compact on $H^{p}$. Indeed,

Take $\left\{f_{n}\right\}_{n} \in B_{H^{p}}$ uniformly converging to 0 on compact subsets of $\mathbb{D}$.

Write $f_{n}=B_{n} \cdot g_{n}$ with $\left|B_{n}^{*}\right|=1$ a.e. and $g_{n} \in H^{p}$ without zeros in $\mathbb{D}$.

The sequence $G_{n}=g_{n}^{p / q}$ is defined and lies in $B_{H^{q}}$.

Up to (enough) subsequences, we may assume that

$$
G_{n} \longrightarrow G \in B_{H^{q}}, \quad B_{n} \longrightarrow B \in B_{H^{\infty}} \quad \text { uniformly on compact subsets of } \mathbb{D}
$$

and the sequence $\left(G_{n} \circ \varphi\right)$ converges to $G \circ \varphi$ in $H^{q}$ (since $C_{\varphi} \in \mathcal{K}\left(H^{q}\right)$ )

$$
\forall z \in \mathbb{D}, \quad\left|f_{n}(z)\right|^{p}=\left|G_{n}(z)\right|^{q}\left|B_{n}(z)\right|^{p} \longrightarrow 0 \text {, hence }|G(z)|^{q}|B(z)|^{p}=0
$$




\section{Compactness}

\section{(Shapiro-Taylor '73) The problem reduces to the hilbertian case:}

$C_{\varphi}$ is compact on $H^{p} \quad$ if and only if $C_{\varphi}$ is compact on $H^{2}$.

If $p, q \geq 1$ and $C_{\varphi}$ is compact on $H^{q}$ then $C_{\varphi}$ is compact on $H^{p}$. Indeed,

Take $\left\{f_{n}\right\}_{n} \in B_{H^{p}}$ uniformly converging to 0 on compact subsets of $\mathbb{D}$.

Write $f_{n}=B_{n} \cdot g_{n}$ with $\left|B_{n}^{*}\right|=1$ a.e. and $g_{n} \in H^{p}$ without zeros in $\mathbb{D}$.

The sequence $G_{n}=g_{n}^{p / q}$ is defined and lies in $B_{H^{q}}$.

Up to (enough) subsequences, we may assume that

$$
G_{n} \longrightarrow G \in B_{H^{q}}, \quad B_{n} \longrightarrow B \in B_{H^{\infty}} \quad \text { uniformly on compact subsets of } \mathbb{D}
$$

and the sequence $\left(G_{n} \circ \varphi\right)$ converges to $G \circ \varphi$ in $H^{q}$ (since $C_{\varphi} \in \mathcal{K}\left(H^{q}\right)$ )

$$
\forall z \in \mathbb{D}, \quad\left|f_{n}(z)\right|^{p}=\left|G_{n}(z)\right|^{q}\left|B_{n}(z)\right|^{p} \longrightarrow 0 \text {, hence }|G(z)|^{q}|B(z)|^{p}=0
$$

Do not forget that $\lambda_{\varphi}(\mathbb{T})=0$

$\int_{\mathbb{T}}\left|f_{n} \circ \varphi^{*}\right|^{p} d \lambda \lesssim \int_{\mathbb{T}}\left|\left(G_{n}-G\right) \circ \varphi^{*}\right|^{q}\left|B_{n} \circ \varphi^{*}\right|^{p} d \lambda+\int_{\mathbb{T}}\left|G \circ \varphi^{*}\right|^{q}\left|B_{n} \circ \varphi^{*}\right|^{p} d \lambda$ 


\section{Compactness}

\section{(Shapiro-Taylor '73) The problem reduces to the hilbertian case:}

$C_{\varphi}$ is compact on $H^{p} \quad$ if and only if $C_{\varphi}$ is compact on $H^{2}$.

If $p, q \geq 1$ and $C_{\varphi}$ is compact on $H^{q}$ then $C_{\varphi}$ is compact on $H^{p}$. Indeed,

Take $\left\{f_{n}\right\}_{n} \in B_{H^{p}}$ uniformly converging to 0 on compact subsets of $\mathbb{D}$.

Write $f_{n}=B_{n} \cdot g_{n}$ with $\left|B_{n}^{*}\right|=1$ a.e. and $g_{n} \in H^{p}$ without zeros in $\mathbb{D}$.

The sequence $G_{n}=g_{n}^{p / q}$ is defined and lies in $B_{H^{q}}$.

Up to (enough) subsequences, we may assume that

$$
G_{n} \longrightarrow G \in B_{H^{q}}, \quad B_{n} \longrightarrow B \in B_{H^{\infty}} \quad \text { uniformly on compact subsets of } \mathbb{D}
$$

and the sequence $\left(G_{n} \circ \varphi\right)$ converges to $G \circ \varphi$ in $H^{q}$ (since $C_{\varphi} \in \mathcal{K}\left(H^{q}\right)$ )

$$
\forall z \in \mathbb{D}, \quad\left|f_{n}(z)\right|^{p}=\left|G_{n}(z)\right|^{q}\left|B_{n}(z)\right|^{p} \longrightarrow 0 \text {, hence }|G(z)|^{q}|B(z)|^{p}=0
$$

Do not forget that $\lambda_{\varphi}(\mathbb{T})=0$

$\int_{\mathbb{T}}\left|f_{n} \circ \varphi^{*}\right|^{p} d \lambda \lesssim \int_{\mathbb{T}}\left|\left(G_{n}-G\right) \circ \varphi^{*}\right|^{q} d \lambda \quad+\int_{\mathbb{T}}\left|G \circ \varphi^{*}\right|^{q}\left|B_{n} \circ \varphi^{*}\right|^{p} d \lambda$ 


\section{Compactness}

\section{(Shapiro-Taylor '73) The problem reduces to the hilbertian case:}

$\mathrm{C}_{\varphi}$ is compact on $\mathrm{H}^{p} \quad$ if and only if $C_{\varphi}$ is compact on $\mathrm{H}^{2}$.

If $p, q \geq 1$ and $C_{\varphi}$ is compact on $H^{q}$ then $C_{\varphi}$ is compact on $H^{p}$. Indeed,

Take $\left\{f_{n}\right\}_{n} \in B_{H^{p}}$ uniformly converging to 0 on compact subsets of $\mathbb{D}$.

Write $f_{n}=B_{n} \cdot g_{n}$ with $\left|B_{n}^{*}\right|=1$ a.e. and $g_{n} \in H^{p}$ without zeros in $\mathbb{D}$.

The sequence $G_{n}=g_{n}^{p / q}$ is defined and lies in $B_{H^{q}}$.

Up to (enough) subsequences, we may assume that

$$
G_{n} \longrightarrow G \in B_{H^{q}}, \quad B_{n} \longrightarrow B \in B_{H^{\infty}} \quad \text { uniformly on compact subsets of } \mathbb{D}
$$

and the sequence $\left(G_{n} \circ \varphi\right)$ converges to $G \circ \varphi$ in $H^{q}$ (since $C_{\varphi} \in \mathcal{K}\left(H^{q}\right)$ )

$$
\forall z \in \mathbb{D}, \quad\left|f_{n}(z)\right|^{p}=\left|G_{n}(z)\right|^{q}\left|B_{n}(z)\right|^{p} \longrightarrow 0 \text {, hence }|G(z)|^{q}|B(z)|^{p}=0
$$

Do not forget that $\lambda_{\varphi}(\mathbb{T})=0$

$\int_{\mathbb{T}}\left|f_{n} \circ \varphi^{*}\right|^{p} d \lambda \lesssim \int_{\mathbb{T}}\left|\left(G_{n}-G\right) \circ \varphi^{*}\right|^{q} d \lambda \quad+\int_{\mathbb{T}}\left|G \circ \varphi^{*}\right|^{q}\left|B_{n} \circ \varphi^{*}\right|^{p} d \lambda$

The dominated convergence theorem gives that the second term converges to 0 . 


\section{Compactness}

\section{(Shapiro-Taylor '73) The problem reduces to the hilbertian case:}

$C_{\varphi}$ is compact on $H^{p} \quad$ if and only if $C_{\varphi}$ is compact on $H^{2}$.

If $p, q \geq 1$ and $C_{\varphi}$ is compact on $H^{q}$ then $C_{\varphi}$ is compact on $H^{p}$. Indeed,

Take $\left\{f_{n}\right\}_{n} \in B_{H^{p}}$ uniformly converging to 0 on compact subsets of $\mathbb{D}$.

Write $f_{n}=B_{n} \cdot g_{n}$ with $\left|B_{n}^{*}\right|=1$ a.e. and $g_{n} \in H^{p}$ without zeros in $\mathbb{D}$.

The sequence $G_{n}=g_{n}^{p / q}$ is defined and lies in $B_{H^{q}}$.

Up to (enough) subsequences, we may assume that

$$
G_{n} \longrightarrow G \in B_{H^{q}}, \quad B_{n} \longrightarrow B \in B_{H^{\infty}} \quad \text { uniformly on compact subsets of } \mathbb{D}
$$

and the sequence $\left(G_{n} \circ \varphi\right)$ converges to $G \circ \varphi$ in $H^{q}$ (since $C_{\varphi} \in \mathcal{K}\left(H^{q}\right)$ )

$$
\forall z \in \mathbb{D}, \quad\left|f_{n}(z)\right|^{p}=\left|G_{n}(z)\right|^{q}\left|B_{n}(z)\right|^{p} \longrightarrow 0 \text {, hence }|G(z)|^{q}|B(z)|^{p}=0
$$

Do not forget that $\lambda_{\varphi}(\mathbb{T})=0$

$\int_{\mathbb{T}}\left|f_{n} \circ \varphi^{*}\right|^{p} d \lambda \lesssim \int_{\mathbb{T}}\left|\left(G_{n}-G\right) \circ \varphi^{*}\right|^{q} d \lambda \quad+\int_{\mathbb{T}}\left|G \circ \varphi^{*}\right|^{q}\left|B_{n} \circ \varphi^{*}\right|^{p} d \lambda$

The dominated convergence theorem gives that the second term converges to 0 . The compactness of $C_{\varphi}$ on $H^{p}$ is proved. 


\section{Compactness}

\section{Definition}

An operator $T: H \rightarrow H$ is Hilbert-Schmidt if for an (any) orthonormal basis $\left(b_{n}\right)$, we have

$$
\|T\|_{H S}^{2}=\sum\left\|T\left(b_{n}\right)\right\|_{H}^{2}<+\infty
$$




\section{Compactness}

\section{Definition}

An operator $T: H \rightarrow H$ is Hilbert-Schmidt if for an (any) orthonormal basis $\left(b_{n}\right)$, we have

$$
\|T\|_{H S}^{2}=\sum\left\|T\left(b_{n}\right)\right\|_{H}^{2}<+\infty
$$

Hilbert-Schmidt operators are compact ! 


\section{Compactness}

\section{Definition}

An operator $T: H \rightarrow H$ is Hilbert-Schmidt if for an (any) orthonormal basis $\left(b_{n}\right)$, we have

$$
\|T\|_{H S}^{2}=\sum\left\|T\left(b_{n}\right)\right\|_{H}^{2}<+\infty
$$

\section{Hilbert-Schmidt operators are compact !}

\section{(Shapiro-Taylor '73) Hilbert-Schmidt composition operators}

$C_{\varphi}$ is Hilbert-Schmidt if and only if $\left\|C_{\varphi}\right\|_{H S}^{2}=\int_{\mathbb{T}} \frac{1}{1-\left|\varphi^{*}\right|^{2}} d \lambda<\infty$. 


\section{Compactness}

\section{Definition}

An operator $T: H \rightarrow H$ is Hilbert-Schmidt if for an (any) orthonormal basis $\left(b_{n}\right)$, we have

$$
\|T\|_{H S}^{2}=\sum\left\|T\left(b_{n}\right)\right\|_{H}^{2}<+\infty
$$

Hilbert-Schmidt operators are compact !

(Shapiro-Taylor '73) Hilbert-Schmidt composition operators

$C_{\varphi}$ is Hilbert-Schmidt if and only if $\left\|C_{\varphi}\right\|_{H S}^{2}=\int_{\mathbb{T}} \frac{1}{1-\left|\varphi^{*}\right|^{2}} d \lambda<\infty$.

The sequence $b_{n}(z)=z^{n}$ (where $\left.n \in \mathbb{N}\right)$ is an orthonormal basis of $H^{2} \ldots$ 


\section{Compactness}

\section{Definition}

An operator $T: H \rightarrow H$ is Hilbert-Schmidt if for an (any) orthonormal basis $\left(b_{n}\right)$, we have

$$
\|T\|_{H S}^{2}=\sum\left\|T\left(b_{n}\right)\right\|_{H}^{2}<+\infty
$$

\section{Hilbert-Schmidt operators are compact !}

\section{(Shapiro-Taylor '73) Hilbert-Schmidt composition operators}

$C_{\varphi}$ is Hilbert-Schmidt if and only if $\left\|C_{\varphi}\right\|_{H S}^{2}=\int_{\mathbb{T}} \frac{1}{1-\left|\varphi^{*}\right|^{2}} d \lambda<\infty$.

The sequence $b_{n}(z)=z^{n}$ (where $n \in \mathbb{N}$ ) is an orthonormal basis of $H^{2} \ldots$ Let us compute

$$
\left\|C_{\varphi}\right\|_{H S}^{2}=\sum_{n=0}^{\infty}\left\|\varphi^{n}\right\|_{H^{2}}^{2}=
$$




\section{Compactness}

\section{Definition}

An operator $T: H \rightarrow H$ is Hilbert-Schmidt if for an (any) orthonormal basis $\left(b_{n}\right)$, we have

$$
\|T\|_{H S}^{2}=\sum\left\|T\left(b_{n}\right)\right\|_{H}^{2}<+\infty
$$

\section{Hilbert-Schmidt operators are compact !}

\section{(Shapiro-Taylor '73) Hilbert-Schmidt composition operators}

$C_{\varphi}$ is Hilbert-Schmidt if and only if $\left\|C_{\varphi}\right\|_{H S}^{2}=\int_{\mathbb{T}} \frac{1}{1-\left|\varphi^{*}\right|^{2}} d \lambda<\infty$.

The sequence $b_{n}(z)=z^{n}$ (where $n \in \mathbb{N}$ ) is an orthonormal basis of $H^{2} \ldots$ Let us compute

$$
\left\|C_{\varphi}\right\|_{H S}^{2}=\sum_{n=0}^{\infty}\left\|\varphi^{n}\right\|_{H^{2}}^{2}=\sum_{n=0}^{\infty} \int_{\mathbb{T}}\left|\varphi^{*}\right|^{2 n} d \lambda=
$$




\section{Compactness}

\section{Definition}

An operator $T: H \rightarrow H$ is Hilbert-Schmidt if for an (any) orthonormal basis $\left(b_{n}\right)$, we have

$$
\|T\|_{H S}^{2}=\sum\left\|T\left(b_{n}\right)\right\|_{H}^{2}<+\infty
$$

\section{Hilbert-Schmidt operators are compact !}

\section{(Shapiro-Taylor '73) Hilbert-Schmidt composition operators}

$C_{\varphi}$ is Hilbert-Schmidt if and only if $\left\|C_{\varphi}\right\|_{H S}^{2}=\int_{\mathbb{T}} \frac{1}{1-\left|\varphi^{*}\right|^{2}} d \lambda<\infty$.

The sequence $b_{n}(z)=z^{n}$ (where $n \in \mathbb{N}$ ) is an orthonormal basis of $H^{2} \ldots$ Let us compute

$$
\left\|C_{\varphi}\right\|_{H S}^{2}=\sum_{n=0}^{\infty}\left\|\varphi^{n}\right\|_{H^{2}}^{2}=\sum_{n=0}^{\infty} \int_{\mathbb{T}}\left|\varphi^{*}\right|^{2 n} d \lambda=\int_{\mathbb{T}} \frac{1}{1-\left|\varphi^{*}\right|^{2}} d \lambda .
$$




\section{Compactness}

\section{Definition}

An operator $T: H \rightarrow H$ is Hilbert-Schmidt if for an (any) orthonormal basis $\left(b_{n}\right)$, we have

$$
\|T\|_{H S}^{2}=\sum\left\|T\left(b_{n}\right)\right\|_{H}^{2}<+\infty
$$

\section{Hilbert-Schmidt operators are compact !}

\section{(Shapiro-Taylor '73) Hilbert-Schmidt composition operators}

$C_{\varphi}$ is Hilbert-Schmidt if and only if $\left\|C_{\varphi}\right\|_{H S}^{2}=\int_{\mathbb{T}} \frac{1}{1-\left|\varphi^{*}\right|^{2}} d \lambda<\infty$.

The sequence $b_{n}(z)=z^{n}$ (where $n \in \mathbb{N}$ ) is an orthonormal basis of $H^{2} \ldots$ Let us compute

$$
\left\|C_{\varphi}\right\|_{H S}^{2}=\sum_{n=0}^{\infty}\left\|\varphi^{n}\right\|_{H^{2}}^{2}=\sum_{n=0}^{\infty} \int_{\mathbb{T}}\left|\varphi^{*}\right|^{2 n} d \lambda=\int_{\mathbb{T}} \frac{1}{1-\left|\varphi^{*}\right|^{2}} d \lambda .
$$

It can be also written

$$
\int_{\overline{\mathbb{D}}} \frac{1}{1-|z|^{2}} d \lambda_{\varphi}
$$

\section{Curso Internacional de Análisis Matemático en Andalucía}




\section{Compactness on Hardy spaces: two examples}

$$
\varphi(z)=\frac{1+z}{2}
$$

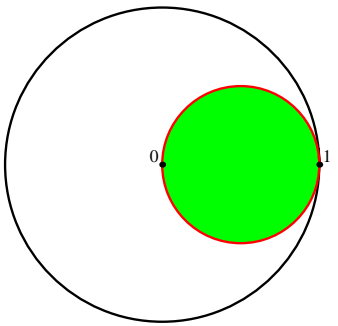




\section{Compactness on Hardy spaces: two examples}

$$
\varphi(z)=\frac{1+z}{2}
$$
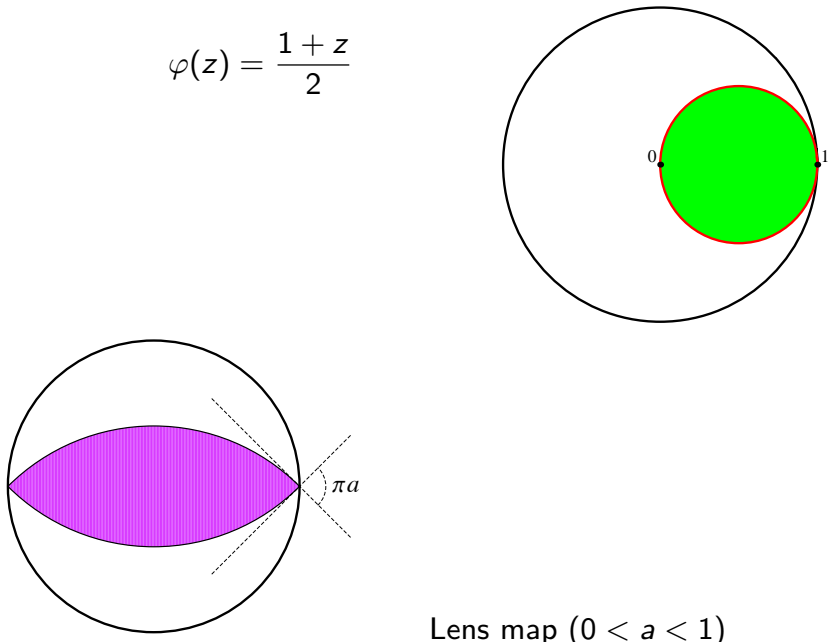

$$
\underline{\text { Lens map }(0<a<1)}
$$

\section{Curso Internacional de Análisis Matemático en Andalucía}




\section{Some characterizations of compactness}

Theorem(Power 80, Mac-Cluer 85)

$C_{\varphi}$ is compact if and only if $\lambda_{\varphi}$ is a vanishing Carleson measure i.e.

$$
\rho_{\varphi}(h)=\sup _{\xi \in \mathbb{T}} \lambda_{\varphi}(W(\xi, h))=o(h) \quad \text { when } h \rightarrow 0
$$




\section{Some characterizations of compactness}

Theorem(Power 80, Mac-Cluer 85)

$C_{\varphi}$ is compact if and only if $\lambda_{\varphi}$ is a vanishing Carleson measure i.e.

$$
\rho_{\varphi}(h)=\sup _{\xi \in \mathbb{T}} \lambda_{\varphi}(W(\xi, h))=o(h) \quad \text { when } h \rightarrow 0
$$

Theorem(Shapiro '87)

$C_{\varphi}$ is compact if and only if $\nu_{\varphi}(h)=\sup _{|w| \geq 1-h} N_{\varphi}(w)=o(h)$ when $h \rightarrow 0$ Actually:

$$
\left\|C_{\varphi}\right\|_{e}=\limsup _{|w| \rightarrow 1^{-}}\left(\frac{N_{\varphi}(w)}{1-|w|}\right)^{1 / 2}=\lim _{h \rightarrow 0}\left(\frac{\nu_{\varphi}(h)}{h}\right)^{1 / 2} .
$$




\section{Some characterizations of compactness}

Theorem(Power 80, Mac-Cluer 85)

$C_{\varphi}$ is compact if and only if $\lambda_{\varphi}$ is a vanishing Carleson measure i.e.

$$
\rho_{\varphi}(h)=\sup _{\xi \in \mathbb{T}} \lambda_{\varphi}(W(\xi, h))=o(h) \quad \text { when } h \rightarrow 0
$$

Theorem(Shapiro '87)

$C_{\varphi}$ is compact if and only if $\nu_{\varphi}(h)=\sup _{|w| \geq 1-h} N_{\varphi}(w)=o(h)$ when $h \rightarrow 0$ Actually:

$$
\left\|C_{\varphi}\right\|_{e}=\limsup _{|w| \rightarrow 1^{-}}\left(\frac{N_{\varphi}(w)}{1-|w|}\right)^{1 / 2}=\lim _{h \rightarrow 0}\left(\frac{\nu_{\varphi}(h)}{h}\right)^{1 / 2} .
$$

(Ackeroyd '10)

$$
\left\|C_{\varphi}\right\|_{e}=\limsup _{|a| \rightarrow 1^{-}}\left\|C_{\varphi}\left(\frac{k_{a}}{\left\|k_{a}\right\|_{H^{2}}}\right)\right\|_{H^{2}}
$$




\section{Some characterizations of compactness}

Let us prove that $C_{\varphi}$ is compact when $\sup _{|w| \geq 1-h} N_{\varphi}(w)=o(h)$ when $h \rightarrow 0$ 


\section{Some characterizations of compactness}

Let us prove that $C_{\varphi}$ is compact when $\sup _{|w| \geq 1-h} N_{\varphi}(w)=o(h)$ when $h \rightarrow 0$

Consider $\left\{f_{n}\right\}_{n} \in B_{H^{2}}$ uniformly converging to 0 on compact subsets of $\mathbb{D}$, 


\section{Some characterizations of compactness}

Let us prove that $C_{\varphi}$ is compact when $\sup _{|w| \geq 1-h} N_{\varphi}(w)=o(h)$ when $h \rightarrow 0$

Consider $\left\{f_{n}\right\}_{n} \in B_{H^{2}}$ uniformly converging to 0 on compact subsets of $\mathbb{D}$, and remember the Littlewood-Paley formula

$$
\left\|f_{n} \circ \varphi\right\|_{2}^{2}=\left|f_{n} \circ \varphi(0)\right|^{2}+2 \int_{\mathbb{D}}\left|f_{n}^{\prime}\right|^{2} N_{\varphi}(z) d \mathcal{A}
$$




\section{Some characterizations of compactness}

Let us prove that $C_{\varphi}$ is compact when $\sup _{|w| \geq 1-h} N_{\varphi}(w)=o(h)$ when $h \rightarrow 0$

Consider $\left\{f_{n}\right\}_{n} \in B_{H^{2}}$ uniformly converging to 0 on compact subsets of $\mathbb{D}$, and remember the Littlewood-Paley formula

$$
\begin{aligned}
\left\|f_{n} \circ \varphi\right\|_{2}^{2} & =\left|f_{n} \circ \varphi(0)\right|^{2}+2 \int_{\mathbb{D}}\left|f_{n}^{\prime}\right|^{2} N_{\varphi}(z) d \mathcal{A} \\
& =\left|f_{n} \circ \varphi(0)\right|^{2}+2 \int_{r \mathbb{D}}\left|f_{n}^{\prime}\right|^{2} N_{\varphi}(z) d \mathcal{A}+2 \int_{\mathbb{D} \backslash r \mathbb{D}}\left|f_{n}^{\prime}\right|^{2} N_{\varphi}(z) d \mathcal{A}
\end{aligned}
$$

for any $r \in(0,1)$.

But 


\section{Some characterizations of compactness}

Let us prove that $C_{\varphi}$ is compact when $\sup _{|w| \geq 1-h} N_{\varphi}(w)=o(h)$ when $h \rightarrow 0$

Consider $\left\{f_{n}\right\}_{n} \in B_{H^{2}}$ uniformly converging to 0 on compact subsets of $\mathbb{D}$, and remember the Littlewood-Paley formula

$$
\begin{aligned}
\left\|f_{n} \circ \varphi\right\|_{2}^{2} & =\left|f_{n} \circ \varphi(0)\right|^{2}+2 \int_{\mathbb{D}}\left|f_{n}^{\prime}\right|^{2} N_{\varphi}(z) d \mathcal{A} \\
& =\left|f_{n} \circ \varphi(0)\right|^{2}+2 \int_{r \mathbb{D}}\left|f_{n}^{\prime}\right|^{2} N_{\varphi}(z) d \mathcal{A}+2 \int_{\mathbb{D} \backslash r \mathbb{D}}\left|f_{n}^{\prime}\right|^{2} N_{\varphi}(z) d \mathcal{A}
\end{aligned}
$$

for any $r \in(0,1)$.

But, fixing $\varepsilon>0$, we may consider some $r \in(0,1)$ (now fixed) such that

$$
\forall z \notin r \mathbb{D}, \quad N_{\varphi}(z) \leq \varepsilon \log (1 /|z|)
$$




\section{Some characterizations of compactness}

Let us prove that $C_{\varphi}$ is compact when $\sup _{|w| \geq 1-h} N_{\varphi}(w)=o(h)$ when $h \rightarrow 0$

Consider $\left\{f_{n}\right\}_{n} \in B_{H^{2}}$ uniformly converging to 0 on compact subsets of $\mathbb{D}$, and remember the Littlewood-Paley formula

$$
\begin{aligned}
\left\|f_{n} \circ \varphi\right\|_{2}^{2} & =\left|f_{n} \circ \varphi(0)\right|^{2}+2 \int_{\mathbb{D}}\left|f_{n}^{\prime}\right|^{2} N_{\varphi}(z) d \mathcal{A} \\
& =\left|f_{n} \circ \varphi(0)\right|^{2}+2 \int_{r \mathbb{D}}\left|f_{n}^{\prime}\right|^{2} N_{\varphi}(z) d \mathcal{A}+2 \int_{\mathbb{D} \backslash r \mathbb{D}}\left|f_{n}^{\prime}\right|^{2} N_{\varphi}(z) d \mathcal{A}
\end{aligned}
$$

for any $r \in(0,1)$.

But, fixing $\varepsilon>0$, we may consider some $r \in(0,1)$ (now fixed) such that

$$
\forall z \notin r \mathbb{D}, \quad N_{\varphi}(z) \leq \varepsilon \log (1 /|z|)
$$

On the other hand, both $f_{n} \circ \varphi(0) \longrightarrow 0$ and $\int_{r \mathbb{D}}\left|f_{n}^{\prime}\right|^{2} N_{\varphi}(z) d \mathcal{A} \longrightarrow 0$. 


\section{Some characterizations of compactness}

Let us prove that $C_{\varphi}$ is compact when $\sup _{|w| \geq 1-h} N_{\varphi}(w)=o(h)$ when $h \rightarrow 0$

Consider $\left\{f_{n}\right\}_{n} \in B_{H^{2}}$ uniformly converging to 0 on compact subsets of $\mathbb{D}$, and remember the Littlewood-Paley formula

$$
\begin{aligned}
\left\|f_{n} \circ \varphi\right\|_{2}^{2} & =\left|f_{n} \circ \varphi(0)\right|^{2}+2 \int_{\mathbb{D}}\left|f_{n}^{\prime}\right|^{2} N_{\varphi}(z) d \mathcal{A} \\
& =\left|f_{n} \circ \varphi(0)\right|^{2}+2 \int_{r \mathbb{D}}\left|f_{n}^{\prime}\right|^{2} N_{\varphi}(z) d \mathcal{A}+2 \int_{\mathbb{D} \backslash r \mathbb{D}}\left|f_{n}^{\prime}\right|^{2} N_{\varphi}(z) d \mathcal{A}
\end{aligned}
$$

for any $r \in(0,1)$.

But, fixing $\varepsilon>0$, we may consider some $r \in(0,1)$ (now fixed) such that

$$
\forall z \notin r \mathbb{D}, \quad N_{\varphi}(z) \leq \varepsilon \log (1 /|z|)
$$

On the other hand, both $f_{n} \circ \varphi(0) \longrightarrow 0$ and $\int_{r \mathbb{D}}\left|f_{n}^{\prime}\right|^{2} N_{\varphi}(z) d \mathcal{A} \longrightarrow 0$.

Hence, for $n$ large enough

$$
\left\|f_{n} \circ \varphi\right\|_{2}^{2} \leq \varepsilon+2 \varepsilon \int_{\mathbb{D} \backslash r \mathbb{D}}\left|f_{n}^{\prime}\right|^{2} \log (1 /|z|) d \mathcal{A}
$$




\section{Some characterizations of compactness}

Let us prove that $C_{\varphi}$ is compact when $\sup _{|w| \geq 1-h} N_{\varphi}(w)=o(h)$ when $h \rightarrow 0$

Consider $\left\{f_{n}\right\}_{n} \in B_{H^{2}}$ uniformly converging to 0 on compact subsets of $\mathbb{D}$, and remember the Littlewood-Paley formula

$$
\begin{aligned}
\left\|f_{n} \circ \varphi\right\|_{2}^{2} & =\left|f_{n} \circ \varphi(0)\right|^{2}+2 \int_{\mathbb{D}}\left|f_{n}^{\prime}\right|^{2} N_{\varphi}(z) d \mathcal{A} \\
& =\left|f_{n} \circ \varphi(0)\right|^{2}+2 \int_{r \mathbb{D}}\left|f_{n}^{\prime}\right|^{2} N_{\varphi}(z) d \mathcal{A}+2 \int_{\mathbb{D} \backslash r \mathbb{D}}\left|f_{n}^{\prime}\right|^{2} N_{\varphi}(z) d \mathcal{A}
\end{aligned}
$$

for any $r \in(0,1)$.

But, fixing $\varepsilon>0$, we may consider some $r \in(0,1)$ (now fixed) such that

$$
\forall z \notin r \mathbb{D}, \quad N_{\varphi}(z) \leq \varepsilon \log (1 /|z|)
$$

On the other hand, both $f_{n} \circ \varphi(0) \longrightarrow 0$ and $\int_{r \mathbb{D}}\left|f_{n}^{\prime}\right|^{2} N_{\varphi}(z) d \mathcal{A} \longrightarrow 0$.

Hence, for $n$ large enough

$$
\left\|f_{n} \circ \varphi\right\|_{2}^{2} \leq \varepsilon+2 \varepsilon \int_{\mathbb{D} \backslash r \mathbb{D}}\left|f_{n}^{\prime}\right|^{2} \log (1 /|z|) d \mathcal{A} \leq \varepsilon+2 \varepsilon\left\|f_{n}\right\|_{2}^{2}=3 \varepsilon .
$$




\section{Some characterizations of compactness}

Let us prove that $C_{\varphi}$ is compact when $\sup _{|w| \geq 1-h} N_{\varphi}(w)=o(h)$ when $h \rightarrow 0$

Consider $\left\{f_{n}\right\}_{n} \in B_{H^{2}}$ uniformly converging to 0 on compact subsets of $\mathbb{D}$, and remember the Littlewood-Paley formula

$$
\begin{aligned}
\left\|f_{n} \circ \varphi\right\|_{2}^{2} & =\left|f_{n} \circ \varphi(0)\right|^{2}+2 \int_{\mathbb{D}}\left|f_{n}^{\prime}\right|^{2} N_{\varphi}(z) d \mathcal{A} \\
& =\left|f_{n} \circ \varphi(0)\right|^{2}+2 \int_{r \mathbb{D}}\left|f_{n}^{\prime}\right|^{2} N_{\varphi}(z) d \mathcal{A}+2 \int_{\mathbb{D} \backslash r \mathbb{D}}\left|f_{n}^{\prime}\right|^{2} N_{\varphi}(z) d \mathcal{A}
\end{aligned}
$$

for any $r \in(0,1)$.

But, fixing $\varepsilon>0$, we may consider some $r \in(0,1)$ (now fixed) such that

$$
\forall z \notin r \mathbb{D}, \quad N_{\varphi}(z) \leq \varepsilon \log (1 /|z|)
$$

On the other hand, both $f_{n} \circ \varphi(0) \longrightarrow 0$ and $\int_{r \mathbb{D}}\left|f_{n}^{\prime}\right|^{2} N_{\varphi}(z) d \mathcal{A} \longrightarrow 0$.

Hence, for $n$ large enough

$$
\left\|f_{n} \circ \varphi\right\|_{2}^{2} \leq \varepsilon+2 \varepsilon \int_{\mathbb{D} \backslash r \mathbb{D}}\left|f_{n}^{\prime}\right|^{2} \log (1 /|z|) d \mathcal{A} \leq \varepsilon+2 \varepsilon\left\|f_{n}\right\|_{2}^{2}=3 \varepsilon .
$$




\section{Back to non-angular derivative}

As a corollary, we get

$$
\begin{gathered}
C_{\varphi} \text { is compact on } H^{p} \Longleftrightarrow \lim _{|z| \rightarrow 1^{-}} \frac{1-|\varphi(z)|}{1-|z|}=\infty \\
\text { if } \varphi \text { univalent (or finitely valent) }
\end{gathered}
$$




\section{Back to non-angular derivative}

As a corollary, we get

$$
\begin{gathered}
C_{\varphi} \text { is compact on } H^{p} \Longleftrightarrow \lim _{|z| \rightarrow 1^{-}} \frac{1-|\varphi(z)|}{1-|z|}=\infty \\
\text { if } \varphi \text { univalent (or finitely valent) }
\end{gathered}
$$

Indeed, if $\varphi$ is $p$-valent:

$$
\frac{N_{\varphi}(w)}{1-|w|} \leq
$$




\section{Back to non-angular derivative}

As a corollary, we get

$$
\begin{gathered}
C_{\varphi} \text { is compact on } H^{p} \Longleftrightarrow \lim _{|z| \rightarrow 1^{-}} \frac{1-|\varphi(z)|}{1-|z|}=\infty \\
\text { if } \varphi \text { univalent (or finitely valent) }
\end{gathered}
$$

Indeed, if $\varphi$ is $p$-valent:

$\frac{N_{\varphi}(w)}{1-|w|} \leq p \frac{\max \{\log (1 /|z|) \mid \varphi(z)=w\}}{1-|w|}$ 


\section{Back to non-angular derivative}

As a corollary, we get

$$
C_{\varphi} \text { is compact on } H^{p} \Longleftrightarrow \lim _{|z| \rightarrow 1^{-}} \frac{1-|\varphi(z)|}{1-|z|}=\infty
$$

if $\varphi$ univalent (or finitely valent)

Indeed, if $\varphi$ is $p$-valent:

$\frac{N_{\varphi}(w)}{1-|w|} \leq p \frac{\max \{\log (1 /|z|) \mid \varphi(z)=w\}}{1-|w|} \approx p \max \left\{\frac{1-|z|}{1-|\varphi(z)|}: \varphi(z)=w\right\}$ 


\section{Back to non-angular derivative}

As a corollary, we get

$$
C_{\varphi} \text { is compact on } H^{p} \Longleftrightarrow \lim _{|z| \rightarrow 1^{-}} \frac{1-|\varphi(z)|}{1-|z|}=\infty
$$

if $\varphi$ univalent (or finitely valent)

Indeed, if $\varphi$ is $p$-valent:

$\frac{N_{\varphi}(w)}{1-|w|} \leq p \frac{\max \{\log (1 /|z|) \mid \varphi(z)=w\}}{1-|w|} \approx p \max \left\{\frac{1-|z|}{1-|\varphi(z)|}: \varphi(z)=w\right\} \longrightarrow 0$ 


\section{Back to non-angular derivative}

As a corollary, we get

$$
\begin{gathered}
C_{\varphi} \text { is compact on } H^{p} \Longleftrightarrow \lim _{|z| \rightarrow 1^{-}} \frac{1-|\varphi(z)|}{1-|z|}=\infty \\
\text { if } \varphi \text { univalent (or finitely valent) }
\end{gathered}
$$

Indeed, if $\varphi$ is $p$-valent:

$$
\frac{N_{\varphi}(w)}{1-|w|} \leq p \frac{\max \{\log (1 /|z|) \mid \varphi(z)=w\}}{1-|w|} \approx p \max \left\{\frac{1-|z|}{1-|\varphi(z)|}: \varphi(z)=w\right\} \longrightarrow 0
$$

The converse is false in general: McCluer-Shapiro ('86) constructed inner functions $\varphi$ admitting no angular derivatives at any point of the circle. 


\section{Angular derivative}

We shall say that $\varphi$ satisfies $(N C)$ if $\lim _{|z| \rightarrow 1^{-}} \frac{1-|\varphi(z)|}{1-|z|}=\infty$. 


\section{Angular derivative}

We shall say that $\varphi$ satisfies $(N C)$ if $\lim _{|z| \rightarrow 1^{-}} \frac{1-|\varphi(z)|}{1-|z|}=\infty$.

We say that $\varphi$ has an angular derivative at $\xi \in \mathbb{T}$, if for some $a \in \mathbb{T}$ the following non-tangential limit exists in $\mathbb{C}$ :

$$
\angle \lim _{z \rightarrow \xi} \frac{\varphi(z)-a}{z-\xi}
$$




\section{Angular derivative}

We shall say that $\varphi$ satisfies $(N C)$ if $\lim _{|z| \rightarrow 1^{-}} \frac{1-|\varphi(z)|}{1-|z|}=\infty$.

We say that $\varphi$ has an angular derivative at $\xi \in \mathbb{T}$, if for some $a \in \mathbb{T}$ the following non-tangential limit exists in $\mathbb{C}$ :

$$
\angle \lim _{z \rightarrow \xi} \frac{\varphi(z)-a}{z-\xi}
$$

Theorem (Julia-Carathéodory)

$\varphi$ satisfies $(N C)$ if and only $\varphi$ has angular derivative at no point $\xi \in \mathbb{T}$. 


\section{Angular derivative}

We shall say that $\varphi$ satisfies $(N C)$ if $\lim _{|z| \rightarrow 1^{-}} \frac{1-|\varphi(z)|}{1-|z|}=\infty$.

We say that $\varphi$ has an angular derivative at $\xi \in \mathbb{T}$, if for some $a \in \mathbb{T}$ the following non-tangential limit exists in $\mathbb{C}$ :

$$
\angle \lim _{z \rightarrow \xi} \frac{\varphi(z)-a}{z-\xi}
$$

Theorem (Julia-Carathéodory)

$\varphi$ satisfies $(N C)$ if and only $\varphi$ has angular derivative at no point $\xi \in \mathbb{T}$.

Observe that, if $\varphi$ has angular derivative at $\xi$ and $a \in \mathbb{T}$ is like in $(A D)$, then

$$
\angle \lim _{z \rightarrow \xi} \varphi(z)=a .
$$




\section{Angular derivative}

We shall say that $\varphi$ satisfies (NC) if $\lim _{|z| \rightarrow 1^{-}} \frac{1-|\varphi(z)|}{1-|z|}=\infty$.

We say that $\varphi$ has an angular derivative at $\xi \in \mathbb{T}$, if for some $a \in \mathbb{T}$ the following non-tangential limit exists in $\mathbb{C}$ :

$$
\angle \lim _{z \rightarrow \xi} \frac{\varphi(z)-a}{z-\xi}
$$

\section{Theorem (Julia-Carathéodory)}

$\varphi$ satisfies $(N C)$ if and only $\varphi$ has angular derivative at no point $\xi \in \mathbb{T}$.

Observe that, if $\varphi$ has angular derivative at $\xi$ and $a \in \mathbb{T}$ is like in $(A D)$, then

$$
\angle \lim _{z \rightarrow \xi} \varphi(z)=a .
$$

This allowed MacCluer and Shapiro ('86) to construct an example of a (finitely valent) symbol $\varphi: \mathbb{D} \rightarrow \mathbb{D}$ such that $C_{\varphi}$ is compact, but $\varphi$ is onto: $\varphi(\mathbb{D})=\mathbb{D}$. 


\section{Construction of the McCluer-Shapiro's example}

Let $g:(0,+\infty) \rightarrow \mathbb{R}$ be a continuous decreasing function such that $\lim _{x \rightarrow 0^{+}} g(x)=+\infty$ (for instance $\left.g(x)=1 / x\right)$. And consider the domain $\Omega=\{x+i y: g(x)<y<g(x)+4 \pi\}$

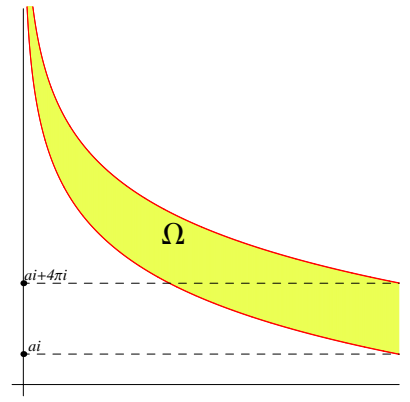

Let $f: \mathbb{D} \rightarrow \Omega$ be a Riemann mapping (a conformal representation) and define 


\section{Construction of the McCluer-Shapiro's example}

Let $g:(0,+\infty) \rightarrow \mathbb{R}$ be a continuous decreasing function such that $\lim _{x \rightarrow 0^{+}} g(x)=+\infty$ (for instance $\left.g(x)=1 / x\right)$. And consider the domain $\Omega=\{x+i y: g(x)<y<g(x)+4 \pi\}$

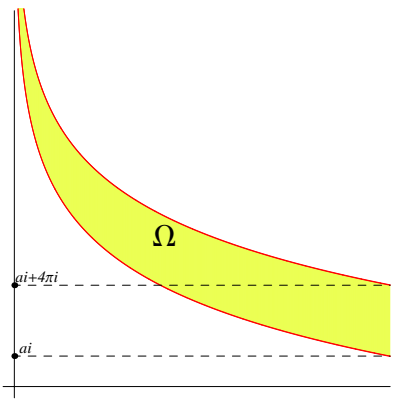

Let $f: \mathbb{D} \rightarrow \Omega$ be a Riemann mapping (a conformal representation) and define

$$
\varphi_{1}(z)=\exp (-f(z)), \quad z \in \mathbb{D} .
$$

and keep in mind that 


\section{Construction of the McCluer-Shapiro's example}

Let $g:(0,+\infty) \rightarrow \mathbb{R}$ be a continuous decreasing function such that $\lim _{x \rightarrow 0^{+}} g(x)=+\infty$ (for instance $\left.g(x)=1 / x\right)$. And consider the domain $\Omega=\{x+i y: g(x)<y<g(x)+4 \pi\}$

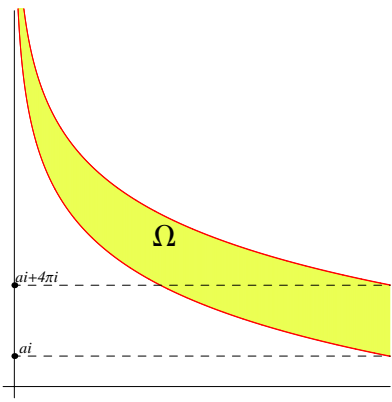

Let $f: \mathbb{D} \rightarrow \Omega$ be a Riemann mapping (a conformal representation) and define

$$
\varphi_{1}(z)=\exp (-f(z)), \quad z \in \mathbb{D} .
$$

and keep in mind that

$$
\left|\varphi_{1}(z)\right| \longrightarrow 1^{-} \Longleftrightarrow \operatorname{Re}(f(z)) \longrightarrow 0^{+}
$$




\section{Angular derivative}

$\varphi_{1}$ is 2 -valent and 


\section{Angular derivative}

$\varphi_{1}$ is 2 -valent and has no point in $\mathbb{T}$ as radial limit (the only way to approach $\mathbb{T}$ is turning and turning inside the disk $\mathbb{D}$ ).

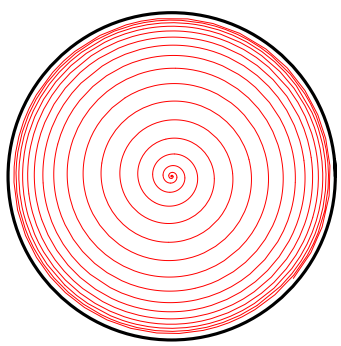




\section{Angular derivative}

$\varphi_{1}$ is 2 -valent and has no point in $\mathbb{T}$ as radial limit (the only way to approach $\mathbb{T}$ is turning and turning inside the disk $\mathbb{D}$ ).

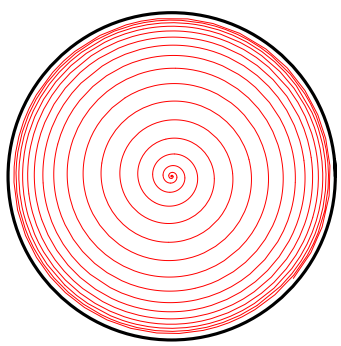

$\varphi_{1}$ is almost onto: $\varphi_{1}(\mathbb{D})=\mathbb{D} \backslash\{0\}$. 


\section{Angular derivative}

$\varphi_{1}$ is 2 -valent and has no point in $\mathbb{T}$ as radial limit (the only way to approach $\mathbb{T}$ is turning and turning inside the disk $\mathbb{D}$ ).

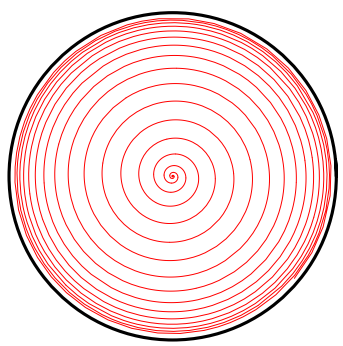

$\varphi_{1}$ is almost onto: $\varphi_{1}(\mathbb{D})=\mathbb{D} \backslash\{0\}$.

Take $a \in \mathbb{D} \backslash\{0\}$ and consider $\varphi=Q_{a} \circ \varphi_{1}$, where $Q_{a}(z)=\left(\frac{a-z}{1-\bar{a} z}\right)^{2}$. 


\section{Angular derivative}

$\varphi_{1}$ is 2 -valent and has no point in $\mathbb{T}$ as radial limit (the only way to approach $\mathbb{T}$ is turning and turning inside the disk $\mathbb{D}$ ).

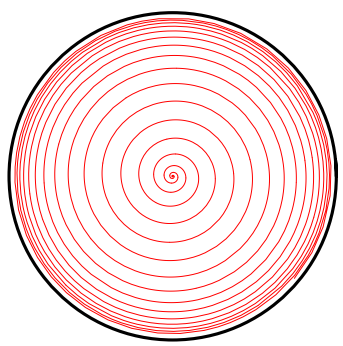

$\varphi_{1}$ is almost onto: $\varphi_{1}(\mathbb{D})=\mathbb{D} \backslash\{0\}$.

Take $a \in \mathbb{D} \backslash\{0\}$ and consider $\varphi=Q_{a} \circ \varphi_{1}$, where $Q_{a}(z)=\left(\frac{a-z}{1-\bar{a} z}\right)^{2}$. $\varphi$ is onto and $C_{\varphi}=C_{\varphi_{1}} \circ C_{Q_{a}}$ is compact. 


\section{Merci !}

